

\title{
Significant Advances in the AIRS Science Team Version-6 Retrieval Algorithm
}

\author{
Joel Susskind ${ }^{1}$, John Blaisdell ${ }^{2}$ and Lena Iredell ${ }^{2}$ \\ ${ }^{1}$ NASA Goddard Space Flight Center, Greenbelt, MD, USA 20771 \\ ${ }^{2}$ SAIC, NASA Goddard Space Flight Center, Greenbelt, MD, USA 20771
}

\begin{abstract}
The Goddard DISC generated products derived from AIRS/AMSU-A observations, starting from September 2002 when the AIRS instrument became stable, using the AIRS Science Team Version-5 retrieval algorithm. The AIRS Science Team Version-6 retrieval algorithm became operational at the Goddard DISC in late 2012. This paper describes some of the significant improvements in retrieval methodology contained in the Version-6 retrieval algorithm, compared to that used in Version-5. In particular, the Science Team made major changes with regard to the algorithms used to 1) derive surface skin temperature and surface spectral emissivity; 2) generate the initial state used to start the cloud clearing and retrieval procedures; and 3) determine Quality Control. This paper describes these advances found in the AIRS Version-6 retrieval algorithm and demonstrates the improvements of some AIRS Version-6 products compared to those obtained using Version-5.
\end{abstract}

Keywords: AIRS/AMSU, high spectral resolution IR sounders, retrieval methodology, IR sounding in cloudy conditions, cloud cleared radiances, Quality Control.

\section{INTRODUCTION}

AIRS was launched on EOS Aqua in May 2002, together with AMSU-A and HSB (which subsequently failed early in the mission), to form a next generation polar orbiting infrared and microwave atmospheric sounding system ${ }^{1}$. AIRS/AMSU had two primary objectives. The first objective was to provide real-time data products available for use by the operational Numerical Weather Prediction Centers in a data assimilation mode to improve the skill of their subsequent forecasts. The second objective was to provide accurate unbiased sounding products with good spatial coverage that are used to generate stable multi-year climate data sets to study the earth's interannual variability, climate processes, and possibly long-term trends.

AIRS is a grating spectrometer with a number of linear arrays of detectors with each detector sensitive to outgoing radiation in a characteristic frequency $v_{i}$ with a spectral band pass $\Delta v_{i}$ of roughly $v_{i} / 1200$. AIRS contains 2378 spectral channels covering portions of the spectral region $650 \mathrm{~cm}^{-1}(15.38 \mu \mathrm{m})-2665 \mathrm{~cm}^{-1}(3.752 \mu \mathrm{m})$. The spectral sampling interval (except for the existence of a few gaps) is $v_{i} / 2400$, giving two AIRS channels per spectral half width. AIRS is accompanied by the temperature sounding $60 \mathrm{GHz}$ microwave instrument AMSU-A. There is a 3x3 array of AIRS footprints within a given AMSU-A footprint, with spatial resolutions of $13 \mathrm{~km}$ and $45 \mathrm{~km}$ at nadir viewing for AIRS and AMSU respectively. Each AIRS footprint is referred to as a Field of View (FOV), and the AMSU-A footprint is referred to as a Field of Regard (FOR).

The Goddard DISC generated products derived from AIRS/AMSU-A observations, starting from September 2002 when the AIRS instrument became stable, using the AIRS Science Team Version-5 retrieval algorithm. ${ }^{2}$ The AIRS Science Team Version-6 retrieval algorithm contains many theoretical and practical improvements over that used in Version-5. The Goddard DISC started processing new AIRS/AMSU data, as well as the reprocessing of all old AIRS/AMSU data, using the AIRS Science Team Version-6 retrieval algorithm in late 2012. The Version-6 retrieval system runs in both in an AIRS/AMSU mode as well as in an "AIRS Only" mode, designed as a back-up system to be used in the event that the AMSU-A instrument continues to degrade significantly beyond its present functionality. This paper describes some of the significant improvements contained in the Version-6 retrieval algorithm, compared to that used in Version-5, with an emphasis on the improvement of ocean surface skin temperatures and atmospheric temperature and water vapor profiles. 
The methodology used to analyze AIRS/AMSU observations is essentially unchanged from that described in Susskind et al. $(2003)^{3}$. Fundamental to this approach is the generation of clear column radiances for each AIRS channel $i, \hat{R}_{i}$, which are derived products representing the radiance channel $i$ would have seen if the entire 3x3 AIRS FOR were cloud free. $\hat{R}_{i}$ is determined for each channel as a linear combination of the observed radiances of that channel in each of the 9 FOV's, using coefficients that are channel independent. The retrieved geophysical state $X$ is subsequently determined which, when substituted in a Radiative Transfer Algorithm (RTA), generates an ensemble of computed radiances $R_{i}(X)$ which are consistent with $\hat{R}_{i}$ for those channels i used in the determination of $X$. Cloud-clearing theory ${ }^{4,5}$ says that to achieve the best results in more stressing cloud conditions, longwave channels sensitive to cloud contamination should be used only in the determination of the coefficients used in the generation of clear column radiances for all channels and not for sounding purposes. In Version- $5^{2}$, tropospheric sounding $15 \mu \mathrm{m} \mathrm{CO} \mathrm{C}_{2}$ observations were used only in the derivation of the cloud clearing coefficients, and temperature profiles were derived using $\hat{R}_{i}$ in the $4.3 \mu \mathrm{m} \mathrm{CO}$ band as well as in some stratospheric $15 \mu \mathrm{m} \mathrm{CO}$ channels that do not see clouds. This new approach allowed for the retrieval of accurate Quality Controlled values of $\hat{R}_{i}$ and $T(p)$ under more stressing cloud conditions than was achievable in Version-4. Version-5 also contained a new methodology to provide accurate case-by-case error estimates for retrieved geophysical parameters and for channel-by-channel clear column radiances. Thresholds of these error estimates were used in a new approach for Quality Control (QC) in Version-5.

The AIRS Version-6 retrieval algorithm has further significant advances over Version-5. The basic theoretical approach used in Version-6 to analyze AIRS/AMSU data is very similar to Version-5 with one major exception. As in Version-5, the coefficients used for generation of cloud cleared radiances $\hat{R}_{i}$ for all channels are determined using observed radiances only in longwave $15 \mu \mathrm{m}$ and $11 \mu \mathrm{m}$ channels. In Version-5, tropospheric temperatures were retrieved using only $\hat{R}_{i}$ in the AIRS shortwave $4.2 \mu \mathrm{m} \mathrm{CO}$ channels, but surface skin temperature was retrieved simultaneously, along with surface spectral emissivity and bi-directional reflectance, using observations both in the longwave 8-12 $\mu \mathrm{m}$ window region and in the shortwave $4.0 \mu \mathrm{m}-3.76 \mu \mathrm{m}$ window region. In Version-6, only window observations in the shortwave window region, $4.0 \mu \mathrm{m}-3.76 \mu \mathrm{m}$, are used to simultaneously determine surface skin temperatures along with shortwave surface spectral emissivities and surface bi-directional reflectance. Longwave surface spectral emissivity is retrieved in a subsequent step. Another significant improvement found in Version-6 is the use of an initial guess $X^{o}$ generated by using Neural-Net methodology ${ }^{6,7}$ in place of the previously used regression approach. These modifications have resulted in significant improvement in the ability to obtain both accurate temperature profiles and surface skin temperatures under more stressing partial cloud cover conditions. The Version-6 retrieval algorithm also has significant further improvements over Version-5 in its Quality Control methodology, as well as other improvements that are not discussed in this paper.

\section{STEPS IN THE VERSION-6 RETRIEVAL ALGORITHM}

Retrievals of all geophysical parameters are physically based and represent states $X_{j, c}$ determined for case $c$ that best match a set of clear column radiances $\hat{R}_{i, c}$ for the subset of AIRS channels $i$ used in the retrieval process. Retrievals of geophysical parameters are performed sequentially, that is, only a subset of the geophysical parameters within the state

$X_{j}$ is modified from that of the incoming state $X_{j}^{0}$ in a given step. A GCM forecast is not used in any way in the retrieval procedure, except for use of the forecasted surface pressure $p_{\text {surf }}$ as the lower pressure boundary when computing expected radiances $R_{i}(X)$ for a given geophysical state $X_{j}$.

In Version- $5^{2}$, the steps in the physical retrieval process were as follows: A regression-based start-up procedure was used to generate the initial state $X^{o}$. Initial clear column radiances $\hat{R}_{k}^{0}$ were generated for all channels $k$ using the initial state $X^{o}$ and cloud-clearing coefficients generated using observed radiances in an ensemble of cloud clearing channels ${ }^{2}$. The state $X^{o}$ is also used as the initial guess to the physical retrieval process in which AIRS/AMSU observations used to retrieve: a) surface skin temperature, surface spectral emissivity and surface bi-directional reflectance of solar radiation; b) atmospheric temperature profile; c) atmospheric moisture profile; d) atmospheric ozone profile; e) atmospheric CO profile; f) atmospheric $\mathrm{CH}_{4}$ profile; and g) cloud properties and OLR. These steps were done sequentially, solving only for the variables to be determined in each retrieval step while using previously determined variables as fixed with an appropriate uncertainty attached to them which was accounted for in the channel noise covariance matrix used in that step. The objective in each step (a-f) was to find solutions which best match $\hat{R}_{i}$ for the subset of channels selected for use 
in that step, bearing in mind the channel noise covariance matrix. Steps a-f were ordered so as to allow for selection of channels in each step which are primarily sensitive to variables to be determined in that step or determined in a previous step, and are relatively insensitive to other parameters. Separation of the problem in this manner allowed for the problem in each step to be made as linear as possible. Step g is performed using the observed radiances $R_{i}$ after the surface and atmospheric conditions have been determined.

In Version-6, there is a slight modification to the sequence of steps used in Version-5, as there is a new step performed in the retrieval sequence. In Version-5, step a) used channels in both the longwave and shortwave window regions and simultaneously solved for surface skin temperature $T_{s}$, shortwave surface spectral emissivity $\varepsilon_{s w}(v)$ and surface spectral bi-directional reflectance $\rho_{s w}(v)$, and longwave surface spectral emissivity $\varepsilon_{\ell w}(v)$. In Version-6, only shortwave window channels are used in this retrieval step to simultaneously determine $T_{s}, \varepsilon_{s w}(v)$, and $\rho_{s w}(v)$. The longwave surface spectral emissivity $\varepsilon_{\ell w}(v)$ is now solved for in a subsequent step using only channels in the longwave window spectral region. This new step is performed after the humidity profile retrieval step because longwave window radiances can be very sensitive to the amount of atmospheric water vapor. The steps used in the Version-6 AO (AIRS Only) algorithm are otherwise identical, but no AMSU-A observations are used in the physical retrieval process or in the generation of the initial state $X^{o}$. In addition, as stated previously, $X^{0}$ is determined in Version-6 via a Neural Net methodology rather than by regression.

\section{CHANNELS AND FUNCTIONS USED IN DIFFERENT STEPS OF VERSION-6}

Figure 1 shows a typical AIRS cloud free brightness temperature spectrum and includes the channels used in Version-6 and Version-6 AO for cloud clearing, as well as in each of the different steps of the AIRS physical retrieval algorithm. These channels are described below.

\subsection{Cloud clearing and temperature profile retrieval}

Following cloud-clearing theory, coefficients needed to generate clear column radiances for all channels are determined using observations in 57 longwave channels, ranging from $701 \mathrm{~cm}^{-1}$ to $1228 \mathrm{~cm}^{-1}$, which we show in yellow in Figure 1 . These same channels are also the ones used in the cloud parameter retrieval step. The temperature profile retrieval step uses 37 channels between $2358 \mathrm{~cm}^{-1}$ and $2395 \mathrm{~cm}^{-1}$ that are sensitive to both stratospheric and tropospheric temperatures, as well as 53 stratospheric sounding channels between $662 \mathrm{~cm}^{-1}$ and $713 \mathrm{~cm}^{-1}$ that are not sensitive to cloud contamination. We show these channels in red in Figure 1. Version-6 also includes 24 additional channels in the temperature profile retrieval step between $2396 \mathrm{~cm}^{-1}$ and $2418 \mathrm{~cm}^{-1}$, also shown in red, that are used in both the temperature profile and the surface parameter retrieval steps. Version-6 includes use of AMSU-A channels 3, 6 and 8-14 in the temperature profile retrieval step as well, while Version-6 AO does not. Version-5 also included AMSU-A channels 4 and 5 in the temperature profile retrieval step, but those channels became noisy in 2007 and are no longer used. AMSU-A channel 7 was noisy at launch and was never used in any step of the retrieval process.

\subsection{Surface skin temperature and longwave spectral emissivity retrievals}

The surface skin temperature retrieval step uses 36 channels between $2420 \mathrm{~cm}^{-1}$ and $2664 \mathrm{~cm}^{-1}$, which we show in light blue in Figure 1, along with the 24 highest frequency channels which are also used in the temperature profile retrieval step. These 60 channels are used to determine the surface skin temperature simultaneously with four independent pieces of information about surface shortwave spectral emissivity and four additional independent pieces of information of shortwave surface bi-directional reflectance during the day. Therefore the skin temperature retrieval steps solves for nine unknowns during the day and five unknowns at night. Surface longwave spectral emissivity is determined using 77 channels, between $758 \mathrm{~cm}^{-1}$ and $1250 \mathrm{~cm}^{-1}$, which we show in purple in Figure 1. In this step, three longwave emissivity perturbation functions are solved for, with $T_{s}$ being held fixed at the value determined from the previous skin temperature retrieval step. The initial guess for surface spectral emissivity in both retrieval steps, $\varepsilon_{v}^{0}$, is set equal to the values found in the AIRS Science Team ocean emissivity model over non-frozen ocean. Over land and frozen ocean, $\varepsilon_{v}^{0}$ is set equal to values coming from the $1^{\circ} \times 1^{\circ}$ monthly mean MODIS Science Team surface spectral emissivity data set. 


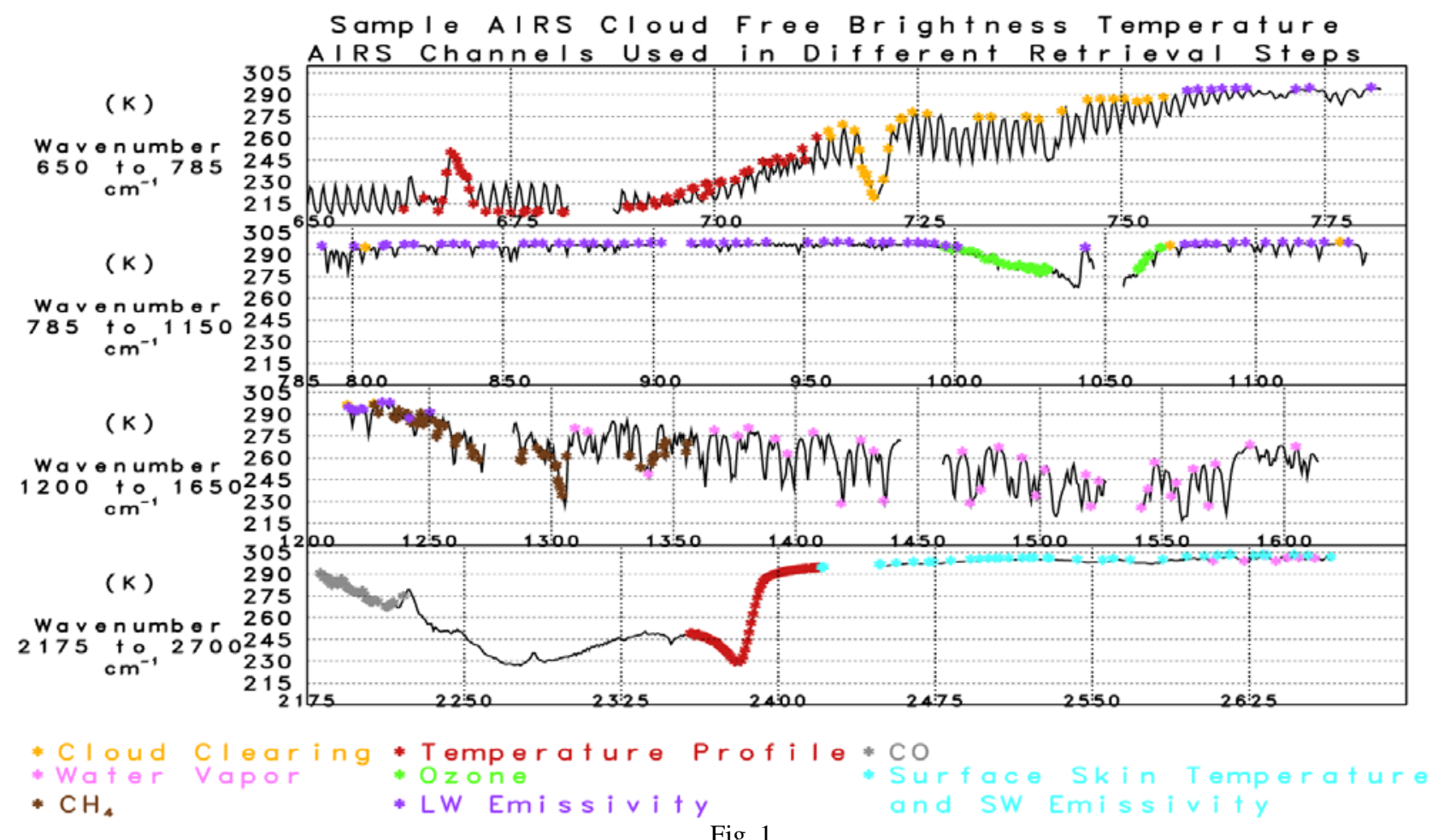

Fig. 1

\subsection{Constituent profile retrievals}

As in Version-5, constituent profile retrievals are performed in separate subsequent steps, each having their own set of channels and functions. Figure 1 shows, in different colors, the Version- 6 channels used in each of these retrieval steps. The $q(p)$ retrieval (pink stars) uses 41 channels in the spectral ranges $1310 \mathrm{~cm}^{-1}$ to $1605 \mathrm{~cm}^{-1}$ and $2608 \mathrm{~cm}^{-1}$ to $2656 \mathrm{~cm}^{-1}$; the $O_{3}(p)$ retrieval (green stars) uses 41 channels between $997 \mathrm{~cm}^{-1}$ and $1069 \mathrm{~cm}^{-1}$; the $C O(p)$ retrieval (gray stars) uses 36 channels between $2181 \mathrm{~cm}^{-1}$ and $2221 \mathrm{~cm}^{-1}$; and the $\mathrm{CH}_{4}(p)$ retrieval (brown stars) uses 58 channels between $1220 \mathrm{~cm}^{-1}$ and $1356 \mathrm{~cm}^{-1}$.

\section{COMPARISON OF QUALITY CONTROLLED VERSION-6 AND VERSION-6 AO RETRIEVALS WITH THOSE OF VERSION-5}

Our evaluation compares V6 and V6 AIRS Only (V6 AO) Quality Controlled products with those of Version-5. In the subsequent sections, we evaluated ocean surface skin temperature $T_{s}$ and surface emissivity $\varepsilon_{v}$, temperature profile $T(p)$, and water vapor profile $q(p)$. Our evaluations compare results obtained on seven focus days to collocated ECMWF truth. The seven focus days are: September 6, 2002; January 25, 2003; September 29, 2004; August 5, 2005; February 24, 2007; August 10, 2007; and May 30, 2010. All products have quality flags based on thresholds of error estimates. Both Version-5 and Version-6 use quality flags for the level-2 output products with the general scheme that QC $=0$ indicates the best quality products designated for use in a data assimilation application. Products flagged with $\mathrm{QC}=1$ are of good quality designated to be included along with those with $\mathrm{QC}=0$ in the generation of gridded Level-3 products used for climate research. Products flagged with $\mathrm{QC}=2$ should not be used for any purpose.

\subsection{Surface skin temperature $\mathrm{T}_{\mathrm{s}}$ and surface spectral emissivity $\varepsilon_{v}$}

Figure 2 shows counts of all Quality Controlled Ocean Surface Skin Temperatures over the latitude range, $50 \mathrm{~N} \quad-50^{\circ} \mathrm{S}$, as a function of the difference between $T_{s}$ and ECMWF "truth" for the 7-day evaluation period. We show the counts of Version-5 retrievals in red and pink, Version-6 retrievals in dark blue and light blue, and Version-6 AO retrievals in black and gray. The lighter shade of each color shows counts of best quality $T_{s}$ retrievals with tight error estimate 
thresholds (QC=0). The darker shade shows counts of both best and good quality $T_{s}$ retrievals including cases with $\mathrm{QC}=0$ or 1, where the error estimate thresholds for $\mathrm{QC}=1$ are looser than those for $\mathrm{QC}=0$.

\section{Surface Skin Temperature Difference (K) 7-Day Daytime and Nighttime Combined} $50^{\circ}$ North to $50^{\circ}$ South Non-Frozen Ocean

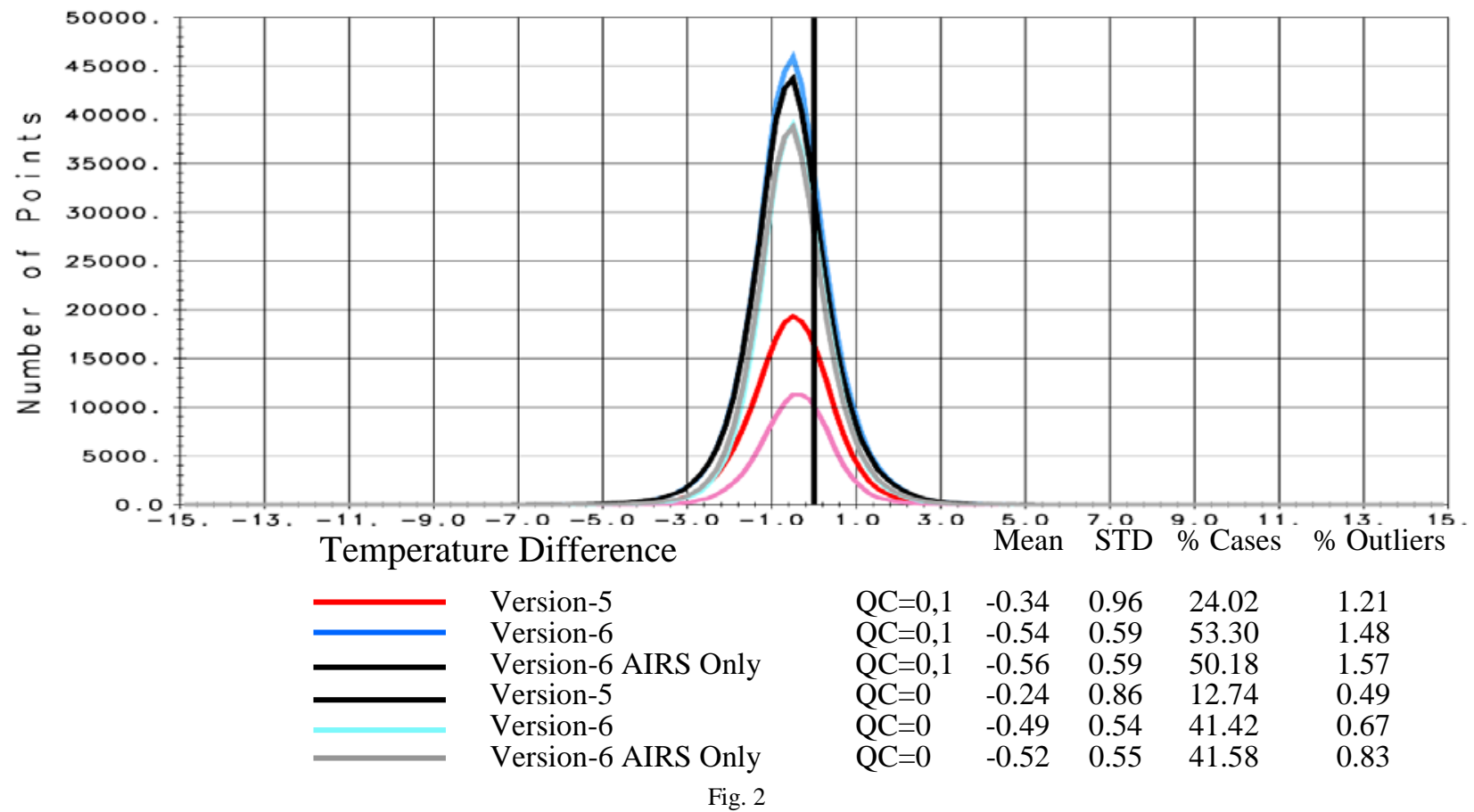

Ocean $T_{s}$ retrievals with QC $=0$ or 1 are the ensemble used to generate the Level-3 Oceanic SST product used for climate studies. Figure 2 also contains statistics for each set of retrievals showing the mean difference from ECMWF, the standard deviation of the ensemble differences, the percentage of all possible cases included in the Quality Controlled ensemble, and percentage of all accepted cases with absolute differences from ECMWF of more than 3K from the mean difference. Such cases are referred to as outliers.

Version-6 QC'd retrievals accept considerably more cases than Version-5 and have much lower standard deviations of the errors as well. In both ensembles, the percentage of outliers grows with loosening the QC thresholds, as expected. Version-6 outliers with QC $=0,1$ are somewhat larger than Version-5, but the yield is more than twice as large. It is noteworthy that Version-6 retrievals with $\mathrm{QC}=0$ have a much smaller percentage of outliers than do Version-5 retrievals with QC $=0,1$ along with a substantially higher yield. One point of slight concern in this figure is that the cold mean bias in Version-6 retrievals compared to ECMWF is somewhat larger than that of Version-5. Statistics of QC'd Version-6 AO retrievals are very similar to those of Version-6.

Figure 3 shows the spatial distribution of the seven day mean differences of the Level-3 oceanic SST products from collocated ECMWF values for both Version-6 and Version-5. The values shown in a given grid box are the average values for that grid box of all cases in which the SST retrieval was accepted either at 1:30 AM or 1:30 PM. The oceanic cases shown in gray indicate where we obtained not a single value of QC'd SST for all 14 possible cases (seven days, twice daily). Figure 3 represents the spatial coverage and accuracy of "pseudo seven day mean” Level-3 products. The seven non-consecutive days included in the figure do not represent a typical seven day Level-3 product, but provide valuable information nonetheless.

Each panel in Figure 3 indicates the mean difference of the Level-3 SST field from its own collocated oceanic ECMWF values, the spatial standard deviation over all grid points of the Level-3 differences from ECMWF, and the percentage of all possible oceanic grid points that have at least one accepted value over the seven day period (i.e., one not gray). The Version-6 Level-3 SST product has much better spatial coverage, with $99.35 \%$ of all oceanic grid points $6 \mathrm{D}-60^{\circ} \mathrm{S}$ 
being filled, compared to Version-5 with only 92.24\%. Moreover, there are large coherent spatial areas in which no Version-5 retrievals were accepted on any of the seven days. The spatial standard deviation of the Version-6 Level-3 SST product errors compared to ECMWF truth is also much smaller than that of Version-5, and the Version-6 area mean negative bias is also smaller than that of Version-5, counter to what might have been expected based on Figure 2.

\section{7-Day Surface Skin Temperature (K) Non-Frozen Ocean Retrieved minus ECMWF AM/PM Average Version-6 \\ Version-5}

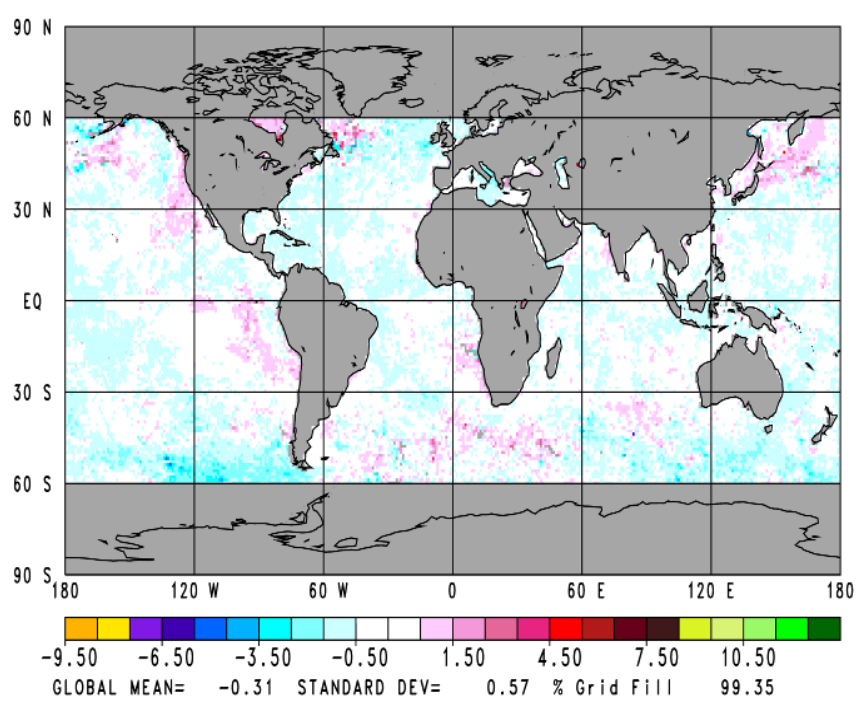

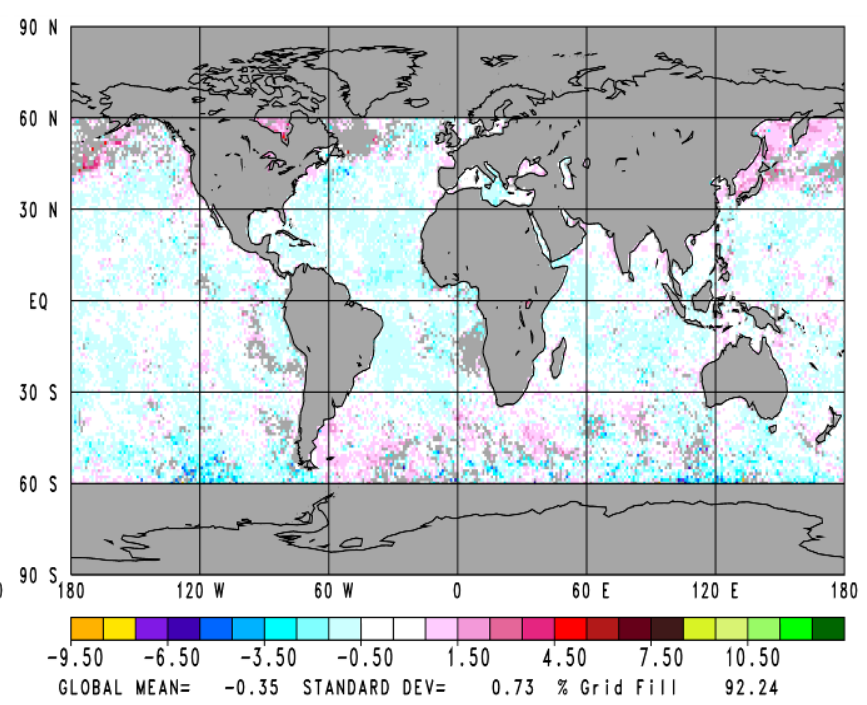

Fig. 3

Figures $4 \mathrm{a}$ and $4 \mathrm{~b}$ show the mean difference of the retrieved ocean surface emissivity $\varepsilon_{v}$ from that of the Masuda model as a function of satellite zenith angle for $v=950 \mathrm{~cm}^{-1}$ and $v=2400 \mathrm{~cm}^{-1}$. The channels are in the longwave and shortwave window regions respectively. In these figures, we show statistics separately for AM orbits in dark colors, and PM orbits in light colors. In both the longwave and shortwave window regions, Version-6 (as well as Version-6 AO) retrieved ocean spectral emissivities as a function of satellite zenith angle are very close to the values expected using the

\section{7-Day Mean Emissivity minus Masuda $50^{\circ}$ North to $50^{\circ}$ South Ocean}
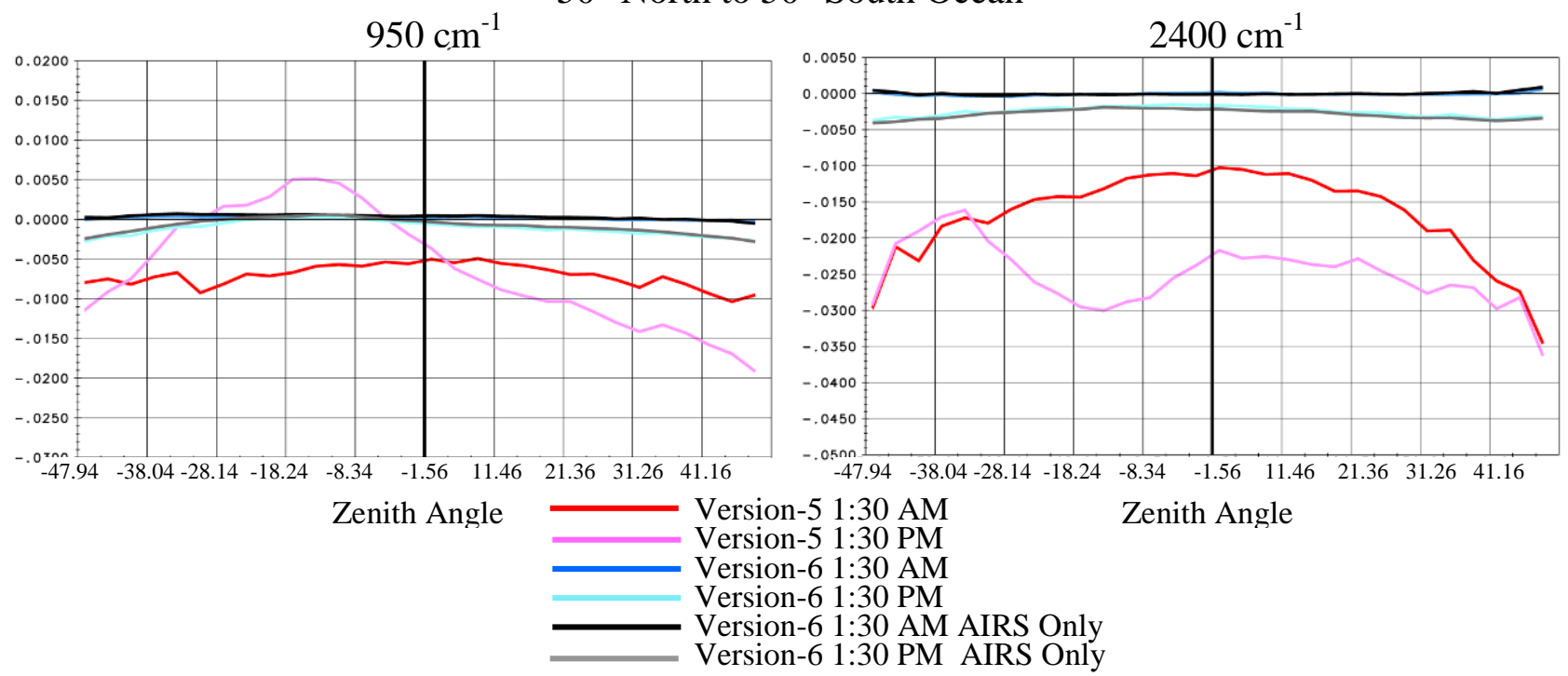

Fig. 4a

Fig. 4b 
Masuda emissivity model. Differences of Version-5 retrieved $\varepsilon_{v}$ from Masuda are much larger than those of Version-6. Version-5 values of $\varepsilon_{v}$ also show a large spurious feature during the day in the vicinity of a satellite zenith angle of -18.24 degrees, which is the viewing angle in which maximum sunglint appears in the field of view. There is no appreciable difference between Version-6 and Version-6 AO in results related to ocean values of $\varepsilon_{v}$.

\section{2 $T(p)$ and $q(p)$ retrieval accuracy as a function of yield}

The fundamentals of the methodology used in Version-6 to retrieve temperature profile $T(p)$ and $q(p)$ from AIRS cloud cleared radiances $\widehat{R}_{\iota}$ are basically the same as those used in Version-5. Quality Controlled (QC'd) Version-6 retrievals of $T(p)$ and $q(p)$ are significantly better than those of Version-5 for three reasons: 1 ) Version-6 uses Neural Net generated first guesses for $T(p)$ and $q(p)$ in place of the regression generated first guess used in Version-5. The Neural Net first guesses are more accurate than the regression guesses, especially under more cloudy conditions. This allows for the generation of accurate QC'd Version-6 retrievals under cloudier cases than was achievable in Version-5; 2) Version-6 has improved QC procedures for $T(p)$ and $q(p)$ than those that were used in Version-5; and 3) Improved Version-6 surface parameters also allow for improved Version-6 $T(p)$ and $q(p)$ in the boundary layer, especially over land.

Figure 5 shows statistics of the differences of QC'd Version-5 and Version-6 retrievals from collocated ECMWF truth for a global ensemble of cases taken over the seven focus days. Panel (a) shows the percentage of QC'd cases accepted as a function of height, panel (b) shows RMS differences of $1 \mathrm{~km}$ layer mean temperatures from collocated ECMWF truth, and panel (c) shows biases of QC'd 1 km layer mean differences from ECMWF. Statistics are shown for six sets of results. We show the results for Version-5 retrievals in red, results for Version-6 retrievals in blue, and results for Version-6 AIRS Only retrievals in black. Version-5 did not have QC'd AIRS Only retrievals. The two sets of curves shown for each experiment represent results using different $T(p)$ QC error estimate thresholds. Version-5 had only one set of QC thresholds, called Standard thresholds. These Version-5 error estimates thresholds were chosen so as to provide a middle ground between the highest accuracy, which would be optimal for Data Assimilation (DA) purposes, and the highest yield (best spatial coverage), which would be optimal for Climate purposes. Experience using Version-5 products showed that Standard QC thresholds were optimal for neither purpose. For example, Data Assimilation experiments using Version-5 retrievals that passed a tighter set of QC thresholds than found in the official Version-5 system resulted in significantly improved forecasts compared to those passing the looser Standard QC thresholds. ${ }^{8}$ The

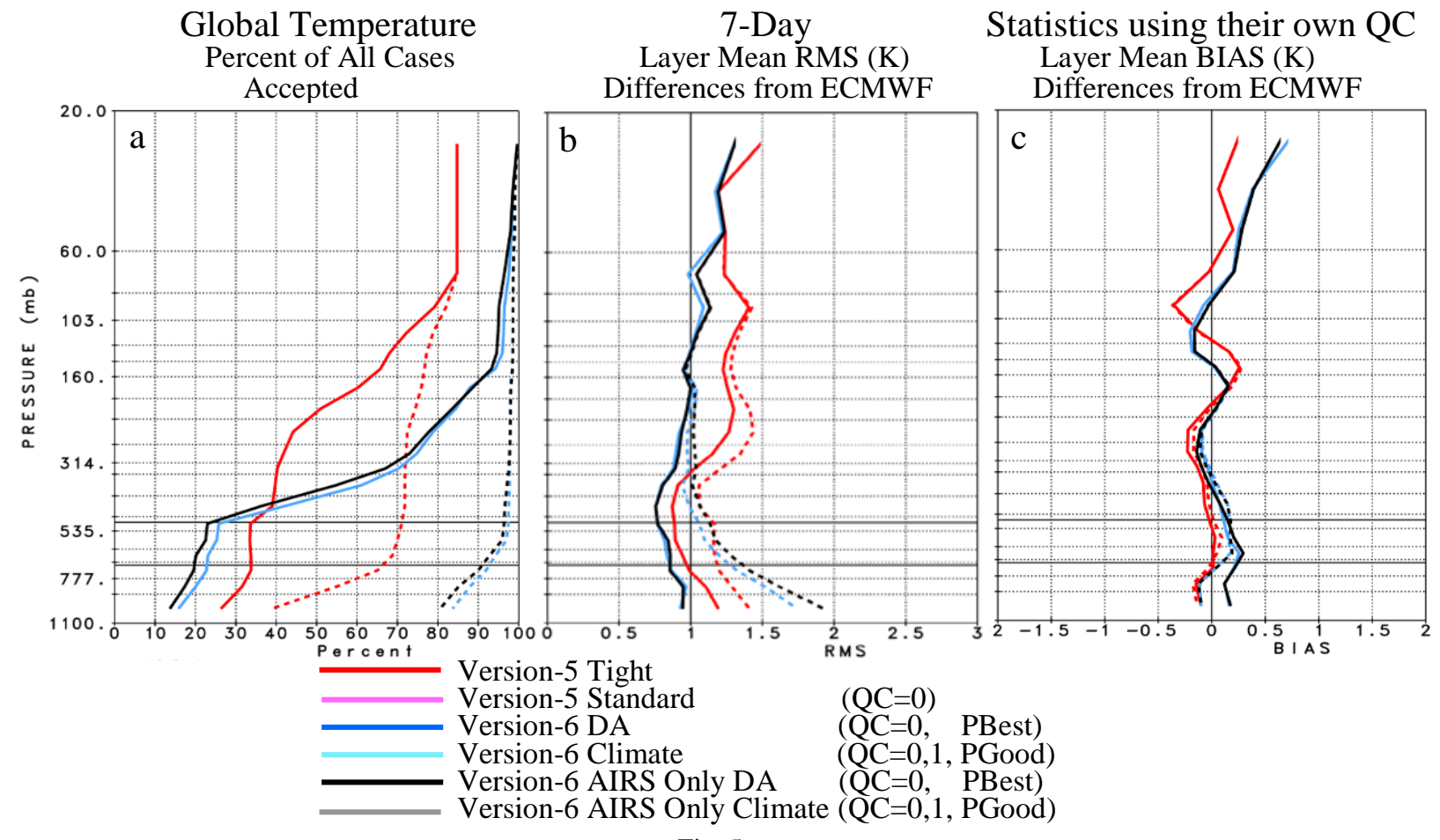

Fig. 5 
solid red lines in Figure 6, and subsequent figures, show statistics of Version-5 retrievals passing the tighter QC thresholds, which we refer to as Tight QC thresholds, and the dashed red lines show equivalent statistics for the ensemble of Version-5 retrievals passing Standard thresholds. Version-6 uses two different sets of thresholds, a very tight set of thresholds newly optimized for Data Assimilation purposes $(\mathrm{QC}=0$ ), and a substantially looser set of thresholds optimal for Climate purposes $(\mathrm{QC}=1)$. As with Version-5, the solid lines show Version-6 and Version-6 AO results using the Data Assimilation (DA) QC thresholds, and the dashed lines show results using the Climate thresholds. Level-3 gridded products utilize all cases passing Climate QC.

In Version-5, all retrievals were either accepted or rejected above $70 \mathrm{mb}$ based on use of different types of tests, even before applying the QC procedures. One of the tests that disqualified the entire temperature profile, and flagged the entire profile with QC=2 (do not use), is that the retrieved cloud fraction is over $90 \%$. Roughly $83 \%$ of Version-5 retrievals passed the initial screening procedure, but none of them occurred in near overcast conditions. Version-5 retrievals with Tight QC have considerably lower yield than those with Standard QC below $200 \mathrm{mb}$, with correspondingly smaller RMS errors, on the order of $1 \mathrm{~K}$ beneath $300 \mathrm{mb}$. There is no appreciable difference in Version-5 bias errors compared to ECMWF found using either set of QC thresholds.

Version-6 does not apply any test which eliminates the entire temperature profile, other than the requirement that the retrieval runs to completion. Version-6 retrievals using DA thresholds have roughly $1 \mathrm{~K}$ RMS errors throughout the atmosphere, with a yield much higher than Version-5 Tight down to about $500 \mathrm{mb}$. Among other benefits from the Data Assimilation perspective, this capability will allow for the assimilation of AIRS temperature products above the clouds in storms, as well as in overcast conditions. The yield of Version-6 retrievals with DA QC is lower than that of Version-5 Tight beneath $500 \mathrm{mb}$, but with a considerable improvement in mid-lower tropospheric temperature RMS errors, with errors less than $1 \mathrm{~K}$, which has been found to be optimal for Data Assimilation purposes. The yield of Version-6 retrievals with Climate QC is extremely high throughout the atmosphere, with a value of about $83 \%$ at the surface. Achievement of this very high yield is extremely valuable in the generation of more representative Level-3 Climate data set. RMS errors of Version-6 retrievals with Climate QC are better than, or comparable to, those of Version-5 with Standard QC down to about $700 \mathrm{mb}$, but with a much higher yield. Beneath $700 \mathrm{mb}$, Version-6 Climate QC RMS errors are somewhat larger than those of Version-5 with Standard QC, but the Version-6 results are essentially unbiased, which is the more important statistic with regard to the generation of the Level-3 products used for Climate research. QC'd results for Version-6 AO are roughly comparable to those of Version-6 but with a somewhat lower yield near the surface.

\section{Global Water Vapor}

Percent Yield

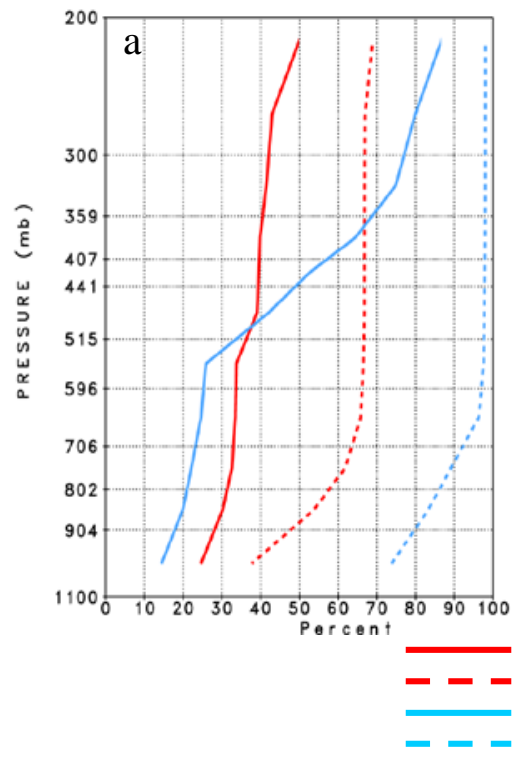

7-Day Statistics use their own QC

$1 \mathrm{Km}$ Layer

Precipitable Water RMS $\%$ Differences from ECMWF

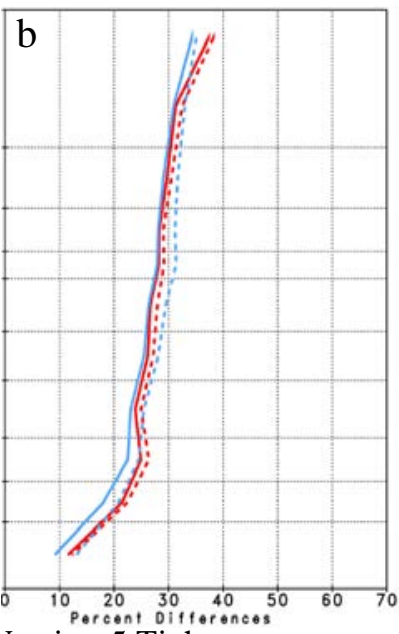

Version-5 Tight

Version-5 Standard

Version-6 DA QC

Version-6 Clim 6
Fig. 6
$1 \mathrm{Km}$ Layer

Precipitable Water Bias \% Differences from ECMWF

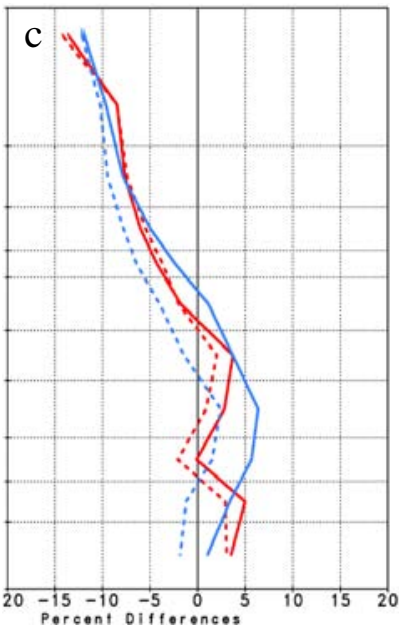

$(\mathrm{QC}=0)$

(QC $=0$ PBest)

QC (QC=0,1 PGood) 
Figure 6 shows analogous results comparing QC'd $1 \mathrm{~km}$ layer precipitable water to that of collocated values of ECMWF. Figure 6 contains results for only Version-5 retrievals and Version- 6 retrievals. We show results only up to $200 \mathrm{mb}$, above which water vapor retrievals are considered of minimal validity, and are not included in the AIRS Science Team Standard Product data set. The relative results regarding Version-5 and Version-6 are analogous to those found for $T(p)$. Version-6 $q(p)$ retrievals with DA QC are considerably improved over those of Version-5 in the lower troposphere. This improvement is at least partially a result of the improved values of $T_{s}$, and $\varepsilon_{v}$ in Version-6 compared to Version-5. As with $T(p)$, Version-6 $q(p)$ retrievals with Climate QC are unbiased, have high accuracy, and contain almost complete spatial coverage.

Figures 5 and 6 provide very important information about the accuracy of Quality Controlled retrievals obtained by different retrieval systems each using their own QC procedures. Indeed, the ability of a different retrieval system to perform QC is a critical part of that retrieval system, especially in the generation of Level-3 products. Figures 5 and 6 do not tell the whole story about the relative accuracy of the retrievals obtained in Version-5 and Version-6, because results are shown for different ensembles of cases. Figure 7a compares RMS $T(p)$ errors of Version- 6 and Version-5 retrievals when evaluated on common ensembles of cases. Results for two such ensembles are shown: an ensemble of relatively easier (less cloudy) cases given by those cases accepted in Version-5 using Tight QC (shown in solid lines); and an ensemble including much more difficult (more cloudy) cases given by those cases accepted in Version-6 using Climate QC, shown in dashed lines. As previously, we show Version-6 RMS errors in blue and Version-5 RMS errors in red.

\section{Global Temperature Layer Mean RMS $\left({ }^{\circ} \mathrm{K}\right)$ Differences from ECMWF}

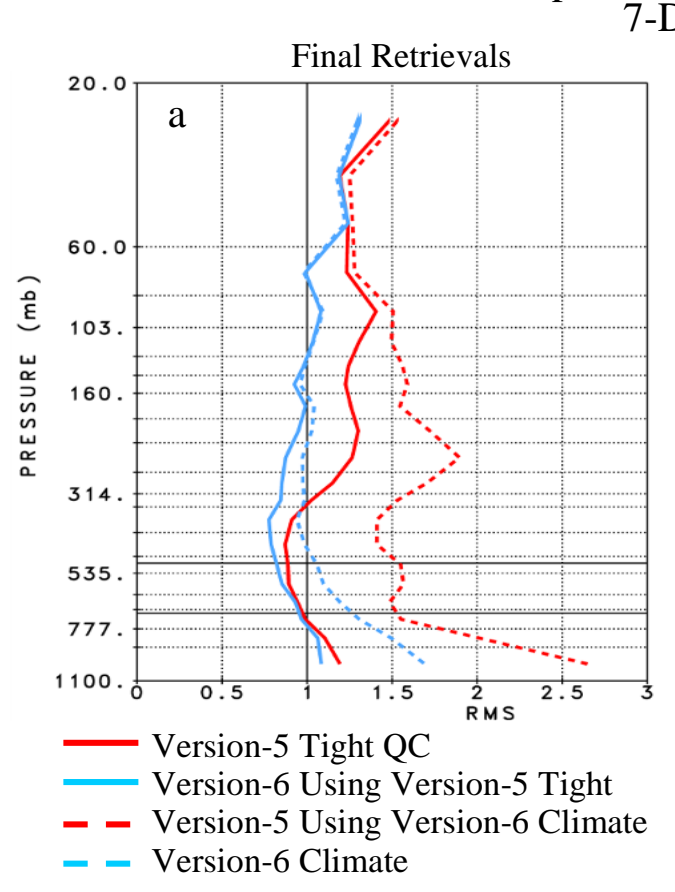
7-Day Two Common Ensembles

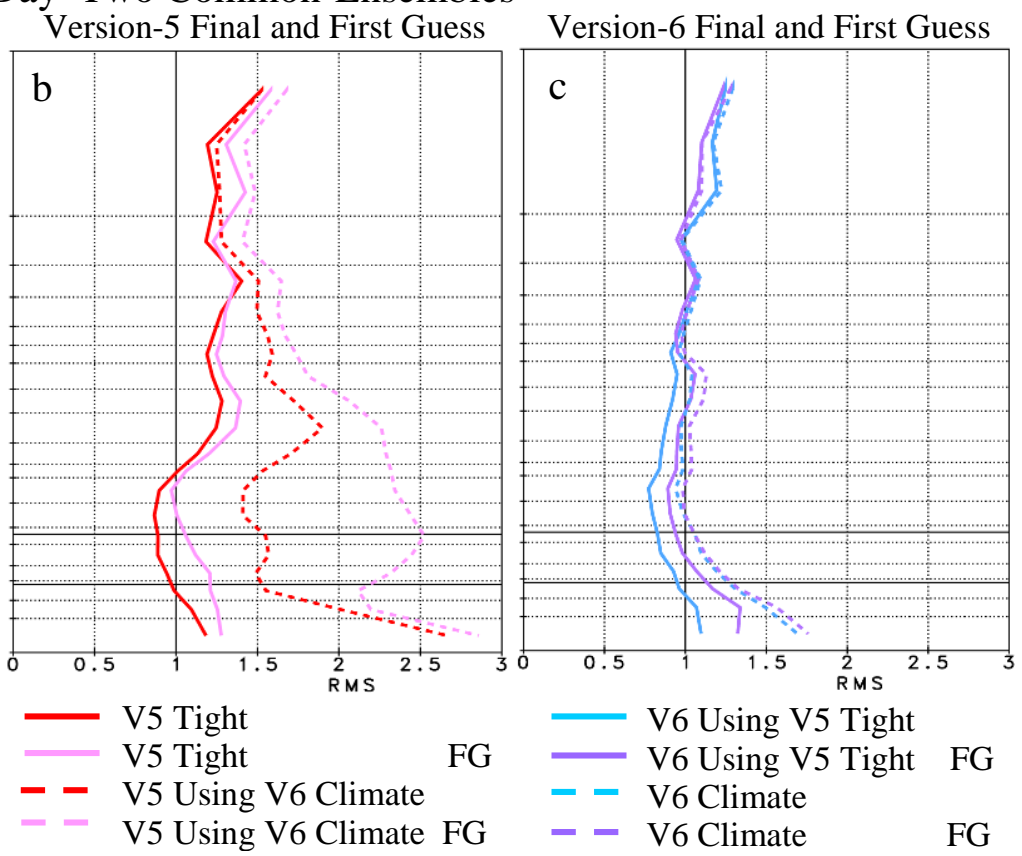

Fig. 7

Version-6 retrievals for the easier (solid line) cases are more accurate than those of Version-5 at all levels, but the degree of improvement below $500 \mathrm{mb}$ is relatively small for these easier cases. The accuracy of Version- 5 retrievals degrades much more rapidly than those of Version-6 for the harder cases (dashed lines). It is for this reason that the Version-5 retrieval system did not use relaxed QC thresholds that would have provided much for higher yields in the generation of Level-3 products from Version-5. Figures 6b and 6c include the same retrieval results shown in Figure 6a, separated into results for Version-5 and Version-6 respectively. Each figure also shows the accuracy of the first guesses used in the appropriate version, and demonstrates the extent that retrievals in a given version improve on their respective first guesses. Both figures show that the physical retrieval improves over the first guess beneath $100 \mathrm{mb}$ on each ensemble of cases. Both sets of first guesses are of comparable quality in the mid-lower troposphere when evaluated on the easier 
ensemble of cases, as are both sets of retrievals. The regression-based first guess degrades much more significantly on the more difficult set of cases than does the Neural-Net first guess. This allows for the ability of Version- 6 to perform so well under almost all cloud conditions. This provides an extremely importaint capability in the generation of highly representative Version-6 Level-3 products used for climate studies.

All results shown so far have been for a global ensemble of cases. Table 1 contains a breakdown of two temperature profile statistics, the Tropospheric Temperature Metric (TTM) and the Boundary Layer Metric (BLM), evaluated over different spatial regions: global; land $50^{\circ} \mathrm{N}$ to $50^{\circ} \mathrm{S}$; ocean $50^{\circ} \mathrm{N}$ to $50^{\circ} \mathrm{S}$; poleward of $50^{\circ} \mathrm{N}$; and poleward of $50^{\circ} \mathrm{S}$. In this table, TTM represents the mean RMS T(p) error over all $1 \mathrm{~km}$ layers from the surface to $100 \mathrm{mb}$, and BLM represents the mean RMS $T(p)$ error over the six lowest $0.25 \mathrm{~km}$ layers from the surface. TTM and BLM results for Version-5 and Version-6 retrievals evaluated over the Version-5 Tight ensemble are shown in Table 1a, and evaluated over the Version6 Climate QC ensemble are given in Table $1 \mathrm{~b}$.

Table 1. 7-Day Mean Statistics Tropospheric Temperature Metric (TTM) and Boundary LayerMetric (BLM)

\section{Cases in Common Using the Version-5 Tight Ensemble}

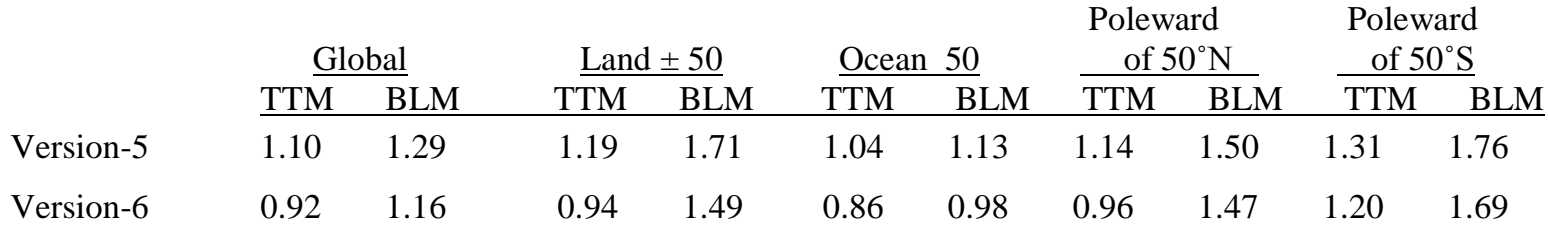

\section{Cases in Common Using the Version-6 Climate Ensemble}

\begin{tabular}{|c|c|c|c|c|c|c|c|c|c|c|}
\hline & \multicolumn{2}{|c|}{ Global } & \multicolumn{2}{|c|}{ Land \pm 50} & \multicolumn{2}{|c|}{ Ocean 50} & \multicolumn{2}{|c|}{$\begin{array}{r}\text { Poleward } \\
\text { of } 50^{\circ} \mathrm{N}\end{array}$} & \multicolumn{2}{|c|}{$\begin{array}{c}\text { Poleward } \\
\text { of } 50^{\circ} \mathrm{S}\end{array}$} \\
\hline & TTM & BLM & TTM & BLM & TTM & BLM & TTM & BLM & TTM & BLM \\
\hline Version-5 & 1.67 & 2.57 & 1.82 & 2.78 & 1.65 & 2.48 & 1.53 & 2.39 & 1.72 & 2.72 \\
\hline Version-6 & 1.11 & 1.67 & 1.06 & 1.75 & 1.03 & 1.34 & 1.12 & 1.93 & 1.32 & 2.02 \\
\hline
\end{tabular}

Version-6 $T(p)$ retrievals are superior to those of Version-5 with regard to both metrics in all spatial regions. It is important to note the improvement of Version-6 Boundary Layer Temperatures compared to Version-5 especially over land, even for the easier ensemble of cases. This improvement over land is at least in part a result of the improved values of surface skin temperature and surface spectral emissivity retrieved over land. A stated goal for Version-6 included improvement of the boundary layer temperature, which has indeed been accomplished. The improvement of boundary layer temperatures is even more pronounced when evaluated over the ensemble of more difficult cases.

Figure 8 shows the spatial distribution of a pseudo-Level-3 seven day field of accepted cases of total precipitable water, $W_{T O T}$, flagged to be of climate quality $(\mathrm{QC}=0,1)$. In Version- $6, W_{T O T}$ is flagged to be of climate quality if the water vapor profile passes the climate QC test down to the surface. Version-5 used a different procedure. The values shown for Version-6 and Version-5 represent the ensemble mean difference, for all accepted cases within that grid box, of the retrieved value of $W_{\text {TOT }}$ from the collocated ECMWF value of $W_{\text {Tот. }}$. We show, in gray, those grid points where we found no accepted values of $W_{\text {TOT }}$ for any of the seven days, either daytime or nighttime. Statistically, Version-6 seven day mean values of $W_{\text {ТОT }}$ are considerably more accurate than those of Version-5, both with regard to global mean bias and as well as to the standard deviation of the errors. Even more important from the climate perspective, spatial coverage of the seven day mean Version-6 product, with $99.89 \%$ of the grid boxes filled, is much more complete than that of Version-5, with $96.12 \%$ of grid boxes filled. Moreover, the $3.88 \%$ of grid boxes for which no accepted soundings were generated in Version-5 tend to come in spatially coherent groups in oceanic areas where low clouds exist. 


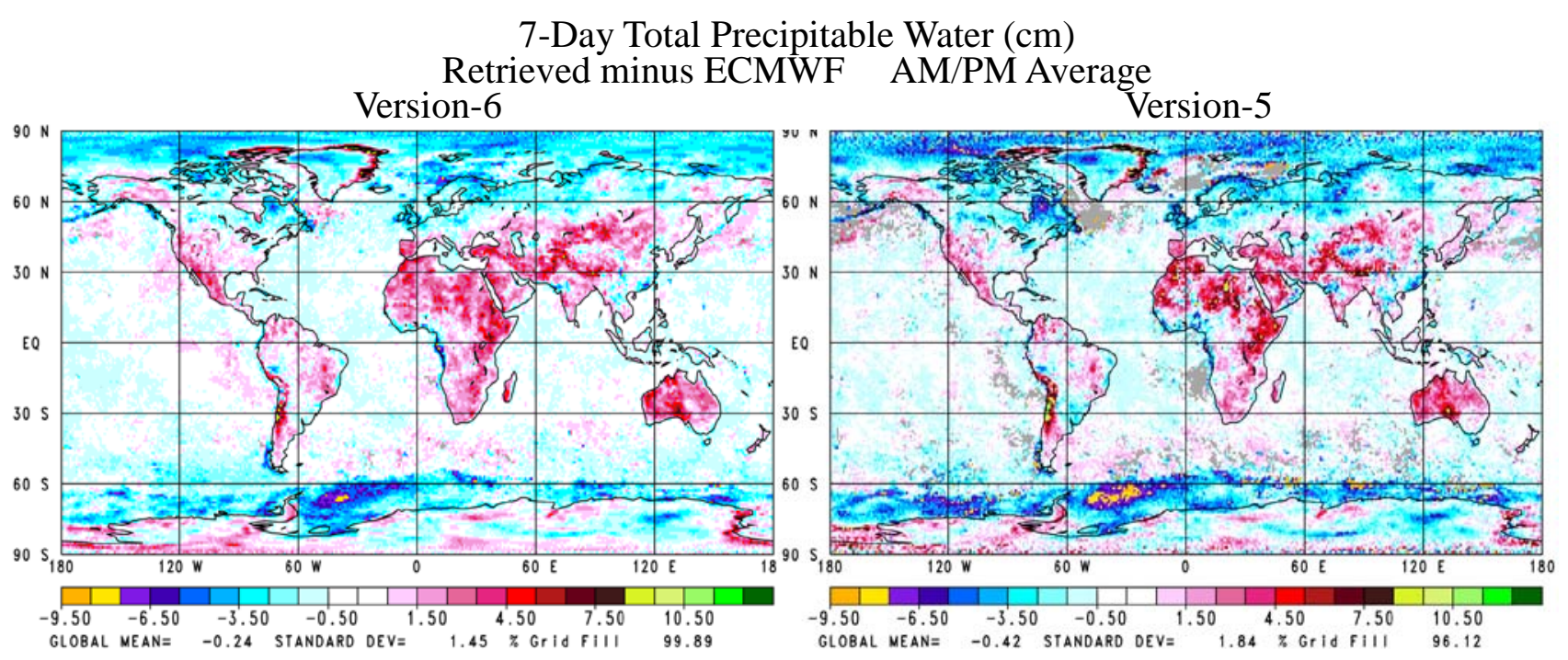

Fig. 8

\subsection{Yield and spurious bias trends of $T(p)$ and $q(p)$}

Version-5 retrievals have two very undesirable characteristics with regard to trends, both of which were considered critical to improve upon in Version-6. The first was that the percent yield of accepted retrievals was found to be decreasing over time (negative yield trend). The second was that the mean differences of QC'd retrieved temperatures from collocated truth values was found to be changing over time as well, especially beneath $300 \mathrm{mb}$ (spurious bias trend). In this section, we examine yield and spurious bias trends of Version-5 and Version-6 products. We computed these trends by taking the slope of the linear least squares fit passing through the values of the appropriate parameter for each of the seven ensemble days as a function of time. Figure 9 shows these trends as a function of pressure for Version5, Version-6, and Version-6 AO. All results show cases with Climate QC (Standard QC for Version-5) because trends are most significant with regard to the generation of Level-3 climate research products. It is apparent that Version- 6

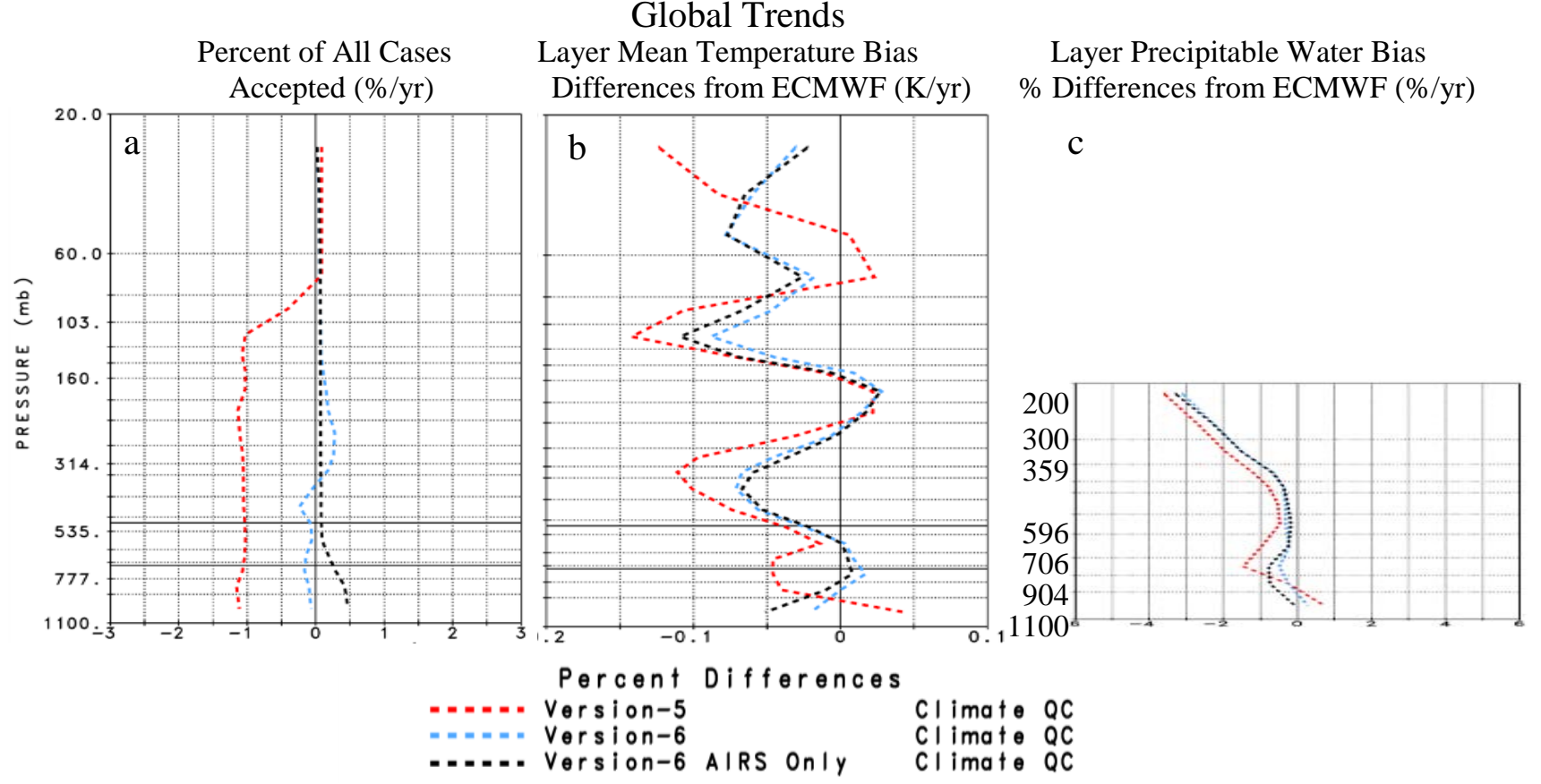

Fig 9 
eliminated the substantial negative tropospheric temperature profile yield trends, on the order of $1 \%$ per year, which were found in Version-5. In addition, the Version-6 negative $T(p)$ bias trends beneath $500 \mathrm{mb}$ are much smaller than those of Version-5, which were on the order of $-0.05 \mathrm{~K} / \mathrm{yr}$. The negative $q(p)$ bias trends found in Version-5 are also substantially reduced in Version-6. It is interesting to note that the spurious $q(p)$ bias trends found in Version-5, as a function of pressure, tend to follow those of $T(p)$ in sign. This is consistent with physics in that a spuriously cold temperature solution for a given case lowers the computed radiance for the water vapor sounding channels in that case. Therefore, if the retrieved temperature for a given case is too low, the solution for $q(p)$ will result in a lowered retrieved water amount as well, so as to raise the radiances computed in the water vapor sounding channels using the incorrect value of $T(p)$ in order to match the cloud cleared radiances for that case. In an analogous fashion, a negative spurious tend of $T(p)$ will result in a spurious negative trend of $q(p)$ as well. Trend results for Version-6 and Version-6 AO are similar to each other.

\section{SUMMARY}

Our validation studies of Version-6 products compared to those of Version-5 indicate that retrieved Version-6 surface skin temperatures and emissivities, as well as temperature and moisture profiles, are superior in every way to those of Version-5. This should enhance their utility both with regard to the study of Climate processes, and also for possible improvement of numerical weather prediction forecast skill when used in a Data Assimilation mode. Accuracy of Version-6 AIRS Only products is only slightly degraded from that of Version-6. This shows that the AIRS Only retrieval system would be adequate to use for Data Assimilation and Climate purposes should AMSU-A fail. This also shows that while it is desirable for an advanced microwave sounder to accompany and advanced IR sounder, it is not a critical component of such a sounding system. This finding is particularly significant towards considerations of the design of an advanced sounding system for flight on Geostationary satellites, on which it is more difficult to build a high spatial resolution microwave sounder.

\section{REFERENCES}

[1] Pagano, T. S., Aumann, H. H., Hagan, D. E. and Overoye, K., "Prelaunch and in-flight radiometric calibration of the Atmospheric Infrared Sounder (AIRS),” IEEE Trans. Geoscience and Remote Sensing, 41, 265-273, DOI: 10.1109/TGRS.2002.808324 (2003).

[2] Susskind, J, Blaisdell, J.M., Iredell, L. and Keita, F., "Improved temperature sounding and quality control methodology using AIRS/AMSU data: The AIRS Science Team Version-5 Retrieval Algorithm,” IEEE Trans. on Geoscience and Remote Sensing, Issue: 99, DOI: 10.1109/TGRS.2010.2070508, 1-15 (2011).

[3] Susskind, J., Barnet, C.D., and Blaisdell, J.M., "Retrieval of atmospheric and surface parameters from AIRS/AMSU/HSB data in the presence of clouds," IEEE Trans. Geoscience and Remote Sensing, 41, 390-409, DOI: 10.1109/TGRS.2002.808236 (2003).

[4] Chahine, M. T., "Remote sensing of cloudy atmospheres. II. Multiple cloud formations,” J. Atmos. Sci., 34, 744-757 (1977).

[5] Chahine, M. T., “Remote sensing of cloudy atmospheres. I. The single cloud layer,” J. Atmos. Sci., 31, 233-243 (1974).

[6] Tao, Z., Blackwell, W.J., Staelin, D.H., "Variance estimation of individual geo-physical parameter retrievals," IEEE Trans. Geoscience and Remote Sensing, submitted for publication (2011).

[7] Blackwell, W.J., "Neural network Jacobian analysis for high-resolution profiling of the atmosphere," EURASIP Journal on Advances in Signal, submitted for publication (2011).

[8] Susskind, J. and Reale, O., "Improving forecast skill by assimilation of AIRS temperature soundings," Geoscience and Remote Sensing Symp. (IGARSS), 2010 IEEE Intl., July 25-30, DOI:10.1109/IGARSS.2010.5652458, 3534-3537 (2010). 


\section{Significant Advances in the AIRS Science Team Version-6 Retrieval Algorithm}

Joel Susskind, John Blaisdell, Lena Iredell

NASA GSFC

Earth Sciences Division - Atmospheres, Code 610

SPIE Optics \& Photonics 2012

Earth Observing Systems XVII

Paper 8510-29

August 15, 2012

San Diego, California 


\section{AIRS/AMSU}

AIRS/AMSU are the advanced IR/MW sounders on EOS Aqua

AIRS has 2360 channels between $650 \mathrm{~cm}^{-1}$ and $2665 \mathrm{~cm}^{-1}$ with $v / \Delta v \approx 1200$

The Goddard DISC has generated products using AIRS Version-5 retrievals starting from September 2002

AIRS Version- 6 has significant improvements compared to Version-5

Version- 6 is completed and will become operational at the DISC in late 2012 for future processing and reprocessing of all AIRS data 


\section{Overview of AIRS/AMSU Version-6 Retrieval Methodology}

We analyze AIRS/AMSU observations using a physically based retrieval system Independent of GCM except for surface pressure - used to compute expected radiances Uses cloud cleared radiances $\hat{\mathrm{R}}_{\mathrm{i}}$ to determine the solution

$\hat{R}_{i}$ represents what AIRS would have seen in the absence of clouds

Derivation of $\hat{R}_{i}$ is updated in different steps of the retrieval process Basic steps

1) Neural-Network initial guess using AIRS/AMSU observations: $X^{0}$

2) Initial cloud clearing produces $\hat{R}_{i}{ }^{0}$ for all channels

Uses $X^{0}$ and observed AIRS radiances

3) Sequentially determine surface parameters, $T(p), q(p), O_{3}(p), C O(p), C H_{4}(p)$, using $\hat{R}_{i}^{0}$

4) Each step uses its own set of channels $\hat{R}_{i}{ }^{0}$

5) Derive cloud parameters and OLR using observed radiances $R_{i}$

Repeat steps 2) - 5)

Generate error estimates and use for Quality Control (QC)

Version- 6 will be run both in AIRS/AMSU and AIRS Only (AO) modes

Version-6 AO retrieval system is analogous but does not use AMSU observations

Provides a back-up system should AMSU fail 


\section{Objectives of AIRS/AMSU}

Provide real time observations to improve numerical weather prediction

Could be $\hat{\mathrm{R}}_{\mathrm{i}}$ (used by NCEP, ECMWF) or $T(p), q(p)$

Accuracy of $T(p), q(p)$ degrades slowly with increasing cloud fraction

There is a trade-off between accuracy and spatial coverage

Using soundings or radiances only in clear cases limits utility of the data

Provide observations to measure and explain interannual variability and trends

Must provide good spatial coverage but also be unbiased

Can be less accurate than needed for data assimilation

Must not contain systematic data gaps in certain regions

AIRS Version-5 and Version- 6 contain accurate error estimates for $T(p), q(p)$, and $\hat{R}_{i}$ Error estimates and quality flags provide options for use in either weather or climate applications 


\section{Improvements in Version-6 Compared to Version-5}

\section{As in Version-5, following theoretical considerations:}

Tropospheric $15 \mu \mathrm{m} \mathrm{CO}$ channels are used only for cloud clearing Gives coefficients to generate clear column radiances $\hat{\mathrm{R}}_{\mathrm{i}}$ for all channels $\widehat{\mathrm{R}}_{\mathrm{i}}$ in $4.3 \mu \mathrm{m} \mathrm{CO}$ channels and stratospheric $15 \mu \mathrm{m} \mathrm{CO} \mathrm{CO}_{2}$ channels are used to determine temperature profile $T(p)$

This allows for accurate temperature profile soundings under more difficult cloud conditions

- The major improvement over Version-5 is the exclusive use of $\hat{R}_{i}$ for shortwave window channels to determine simultaneously $T_{s}$, shortwave spectral emissivity $\varepsilon_{s w}(v)$, and spectral surface bidirectional reflectance $\varepsilon_{s w}(v)$

$\hat{R}_{\mathrm{i}}$ in longwave window channels are used in a subsequent retrieval step to determine $\varepsilon_{\mathrm{LW}}(\mathrm{v})$ given $\mathrm{T}_{\mathrm{s}}$

Version-5 solved for surface skin temperature using $\hat{R}_{i}$ in both longwave and shortwave window channels simultaneously

- Version-6 uses a Neural-Net initial guess while Version-5 used regression guess

The Neural-Net initial guess degrades more slowly than regression with increasing cloud cover

- Version-6 also has further improved the methodology for Quality Control of $T(p), q(p)$

- Version-6 has improved the algorithms used to compute OLR, cloud products, and trace gasses - not discussed today 
Sample AlRS Cloud Free Brightness Temperature AlRS Channels Used in Different Retrieval Steps

(K) $\quad 290$

Wavenumber 260

650 to 785245

$\mathrm{cm}^{-1} 230$

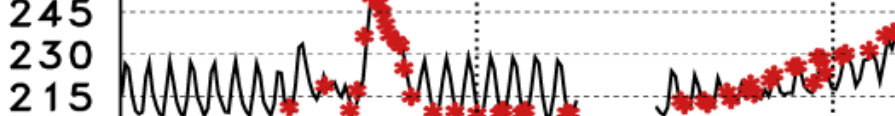

$305^{60}$.

(K)

290

275

Wavenumber 260

785 to 1150245

$\mathrm{cm}^{-1}$

230

305

(K)

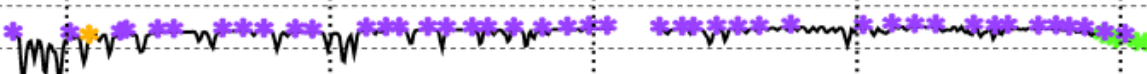
775

Wavenumber 260

1200 to 1650245 $\mathrm{cm}^{-1}$

235
215

$85 \quad 800$ $8: 50$ 900 $9: 50$ 1000 $10 \div 50$

N

Wavenumber 260

$2175+o 2700245$ $\mathrm{cm}^{-1}$

230

2175

$23: 25$

2400

* Cloud CI ear

$* \mathrm{CH}_{4}$
* Temperature Profile*CO

* Ozone

* LW Emiss i vi ty
2475

$25: 50$

2625

$15 \div 50$

$15: 00 \quad 15: 50 \quad 1600$

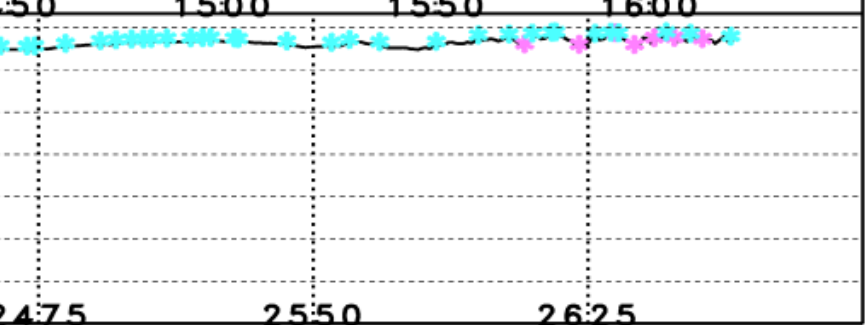

$$
\text { co }
$$

and SW Emissivity 


\section{Methodology Used for Version-5 T(p) Quality Control}

Cases are rejected outright if cloud fraction $>90 \%$. Otherwise:

Define a profile dependent pressure, $\mathrm{p}_{\text {best }}$, above which the temperature profile is flagged as good - beneath $p_{\text {best }}$ profile is flagged as bad

Use error estimate $\delta T(p)$ to determine $p_{\text {best }}$ based on comparison with error estimate thresholds $\Delta \mathrm{T}(\mathrm{p})$

$$
\delta T(p) \leq \Delta T(p) \text { down to } P_{\text {best }}
$$

Temperature profile statistics include errors of $T(p)$ down to $p=p_{\text {best }}$

Version-5 used Standard $\Delta T(p)$ thresholds optimized simultaneously for weather and climate applications - middle ground between accuracy and spatial coverage

Subsequent research has shown that use of more stringent (Tight) $\Delta T(p)$ thresholds to define $p_{\text {best }}$ performs better in Data Assimilation experiments in which $T(p)$ is assimilated down to $p_{\text {best }}$

Tight thresholds gives higher accuracy retrievals with less spatial coverage

Additional research also has shown that looser thresholds (greater spatial coverage) is better for climate applications 


\section{Methodology Used for T(p) Quality Control in Version-6}

Essentially no retrievals are rejected outright unless retrieval fails

All successful retrievals are accepted down to $30 \mathrm{mb}$

$\mathrm{QC}$ is otherwise analogous to Version-5 but uses tight thresholds $\Delta \mathrm{T}_{\mathrm{A}}(\mathrm{p})$ for Data Assimilation and loose thresholds $\Delta T_{C}(p)$ for Climate applications

$\Delta \mathrm{T}_{\mathrm{A}} \mathrm{QC}$ thresholds define $\mathrm{p}_{\text {best }}$ and $\Delta \mathrm{T}_{\mathrm{C}}$ thresholds define $\mathrm{p}_{\text {good }}$

$\mathrm{QC}=0$ down to $\mathrm{p}_{\text {best }}, \mathrm{QC}=1$ between $\mathrm{p}_{\text {best }}$ and $\mathrm{p}_{\text {good }}$ and $\mathrm{QC}=2$ below $\mathrm{p}_{\text {good }}$

Data Assimilation experiments use $T(p)$ down to $p_{\text {best }}$

Climate applications use $T(p)$ down to $p_{\text {good }}$

Level-3 gridded products use cases with $\mathrm{QC}=0$ or 1

$\Delta \mathrm{T}_{\mathrm{A}} \mathrm{QC}$ thresholds were set so as to give RMS errors $\approx 1 \mathrm{~K}$ for accepted cases

$\Delta \mathrm{T}_{\mathrm{C}} \mathrm{QC}$ thresholds were set so as to maximize spatial coverage and achieve $<2 \mathrm{~K}$ tropospheric RMS errors with essentially unbiased retrievals

Results shown are based on retrievals on seven different days in different months and years, using ECMWF as "truth" 


\section{Surface Skin Temperature Difference 7-Day Daytime and Nighttime combined $50^{\circ} \mathrm{N}$ to $50^{\circ} \mathrm{S}$ Non-Frozen Ocean}

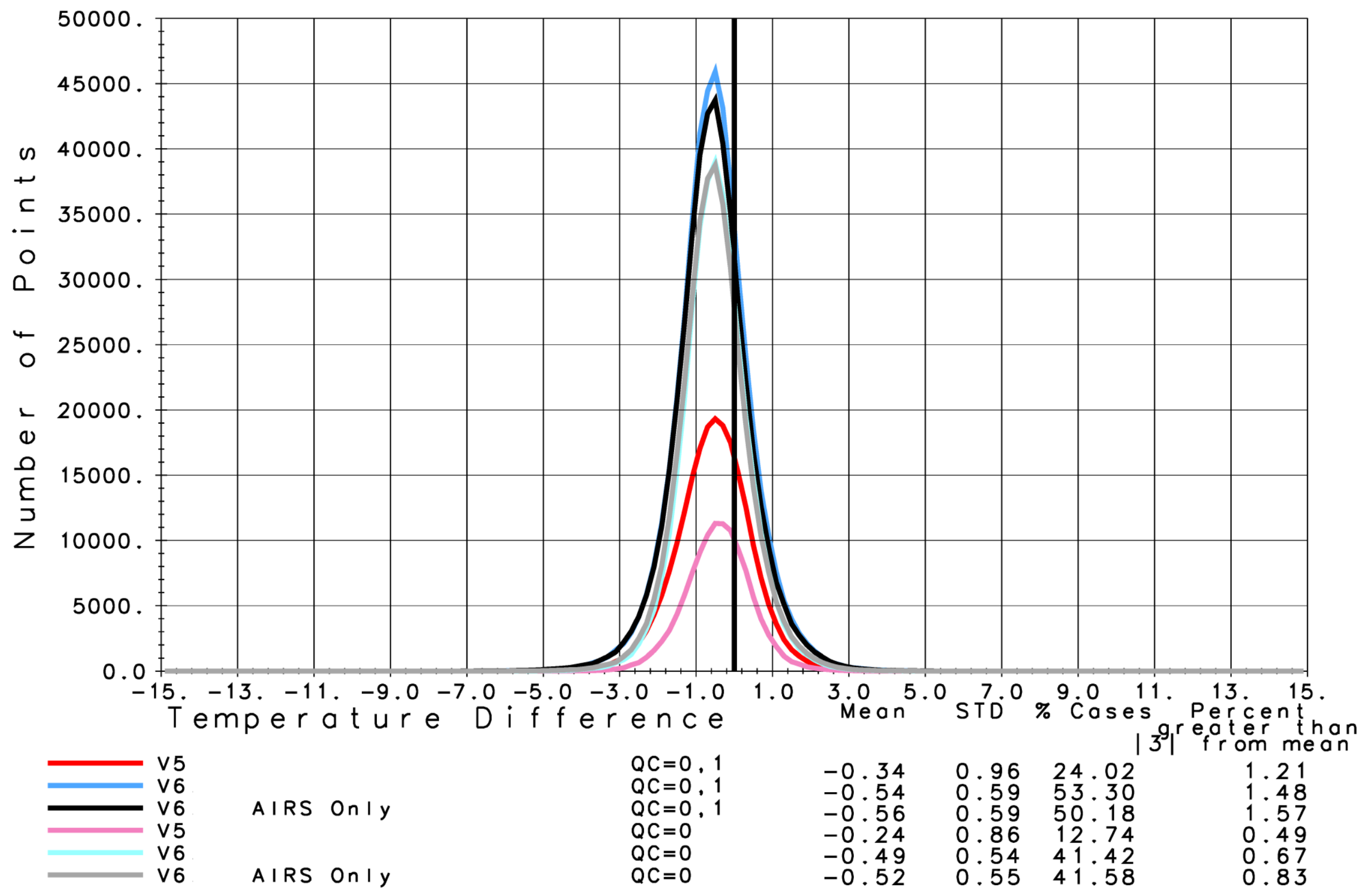




\section{7-Day Mean Emissivity minus Masuda}

$50^{\circ}$ North to $50^{\circ}$ South Ocean

$950 \mathrm{~cm}^{-1}$

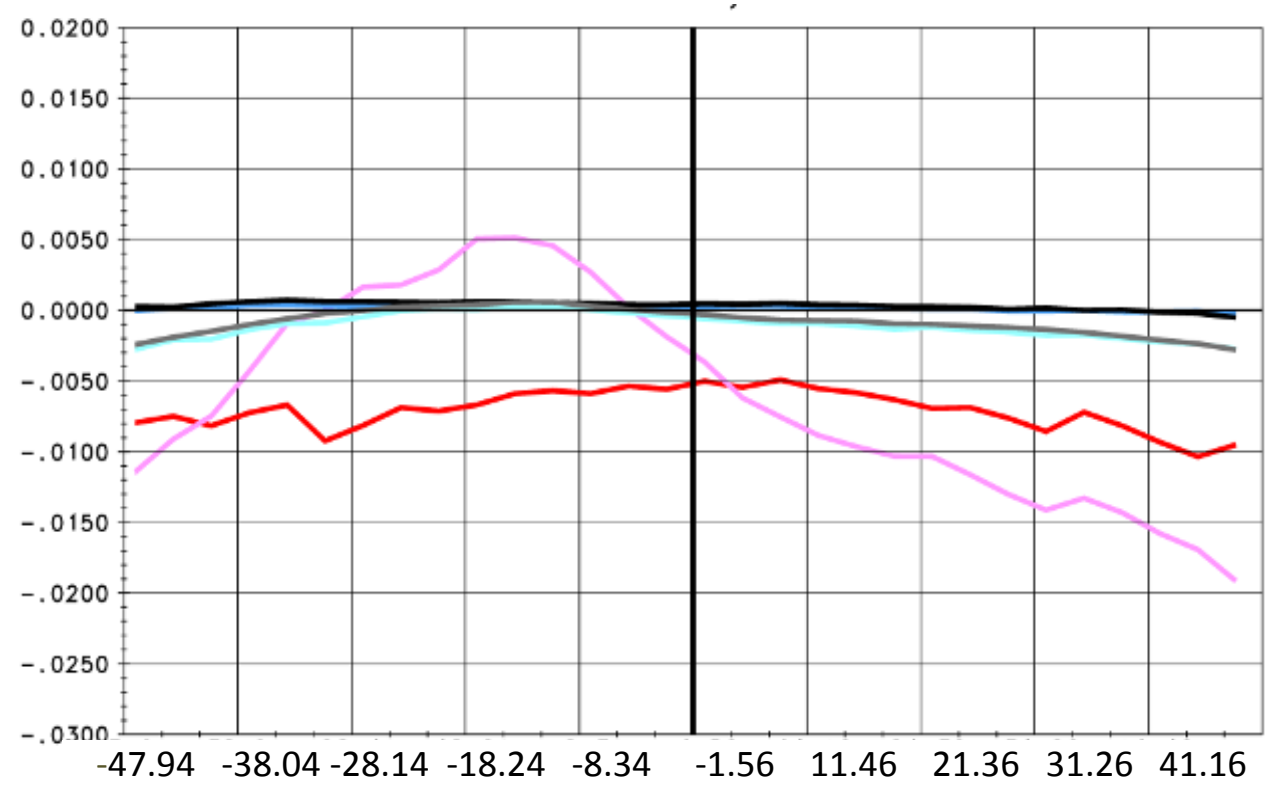

Zenith Angle
$2400 \mathrm{~cm}^{-1}$

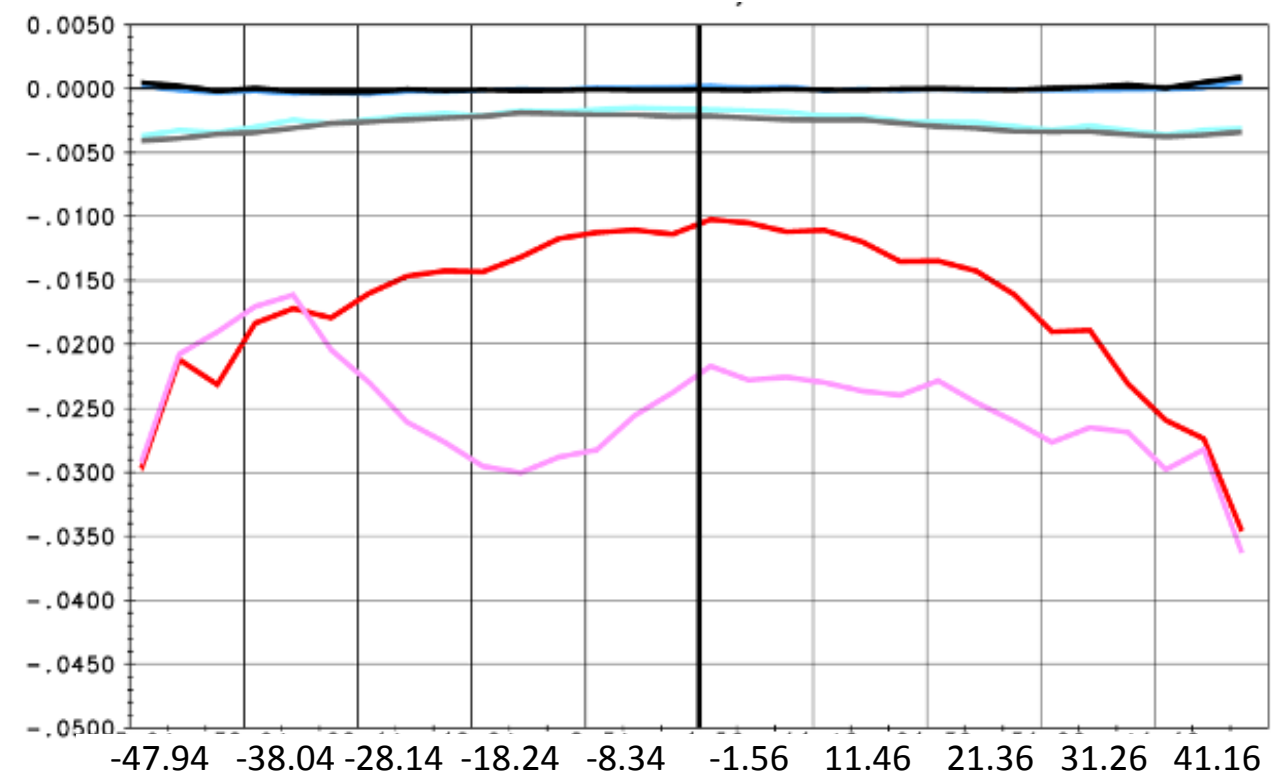

Zenith Angle

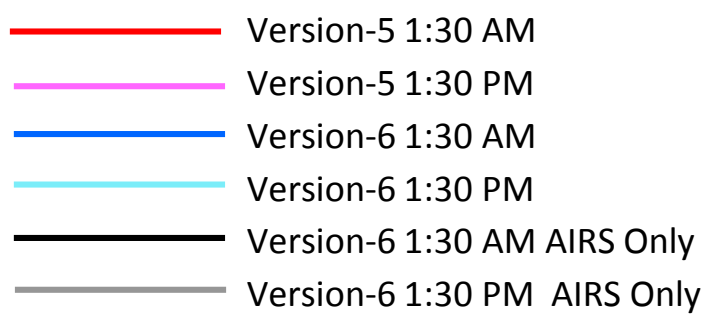

Version-6 Ocean surface emissivities are much closer to Masuda model used as truth Version-5 Ocean surface emissivities are strongly impacted by sunglint during the day 


\section{7-Day Average Surface Skin Temperature (K) Non-Frozen Ocean Retrieved minus ECMWF AM/PM Average}
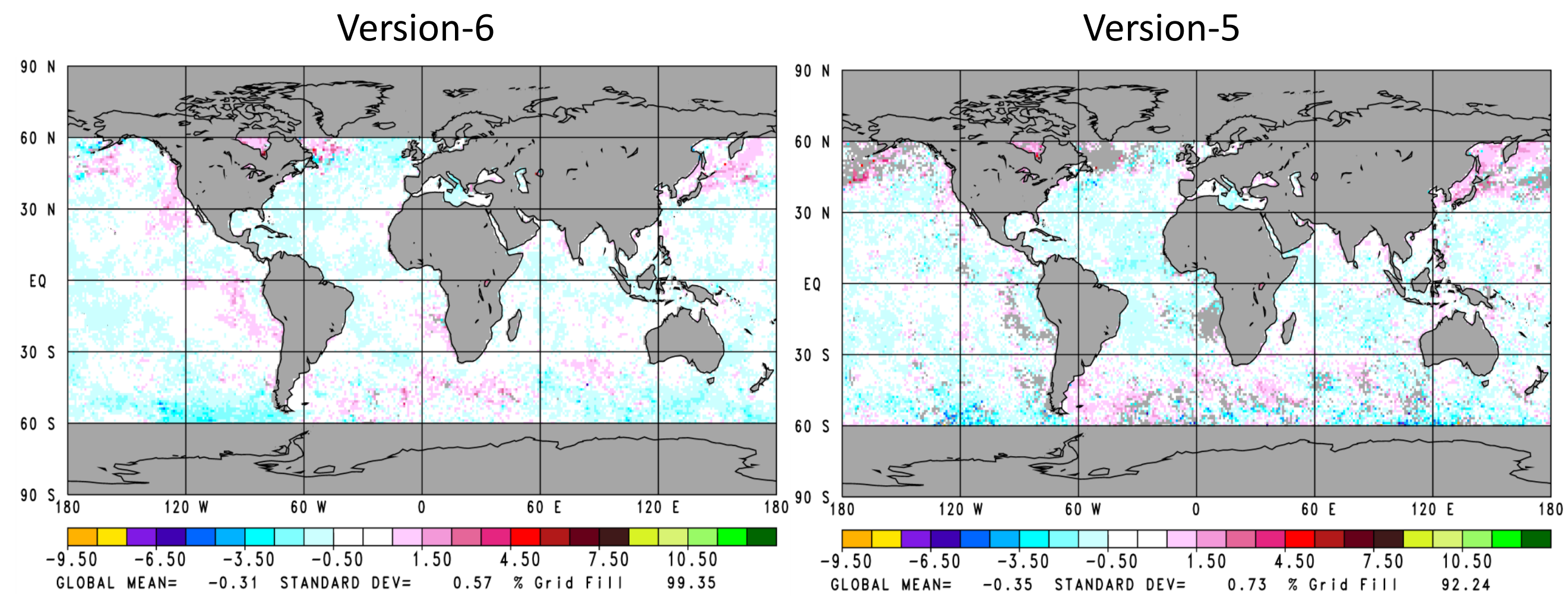

Version-6 7-day mean Level-3 SST product has much better spatial coverage and accuracy than does Version-5, which has gaps in cloudy areas. 


\section{7-Day Total Precipitable Water (cm)}

\section{Retrieved minus ECMWF AM/PM Average}
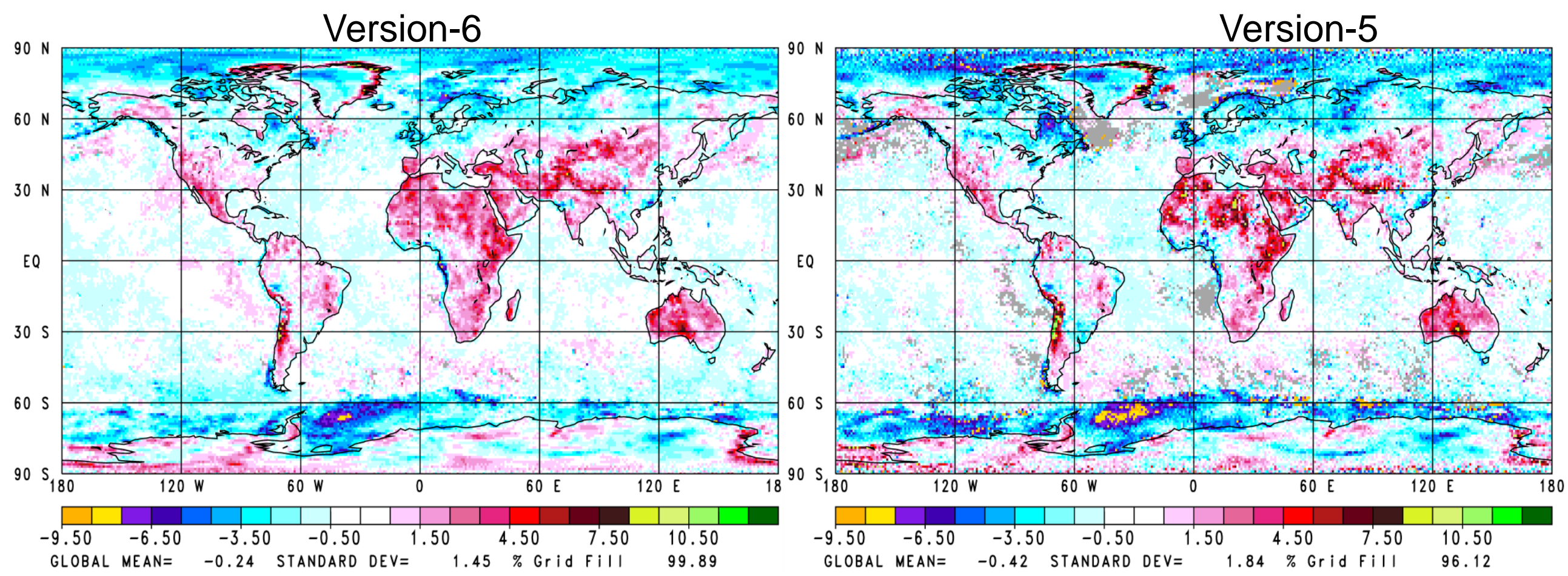

As with Ocean SST, Version-6 7-day mean Total Precipitable Water has much better accuracy and spatial coverage than Version-5 


\section{Global 7-Day Temperature Profile Statistics}

Percent of All Cases

Accepted

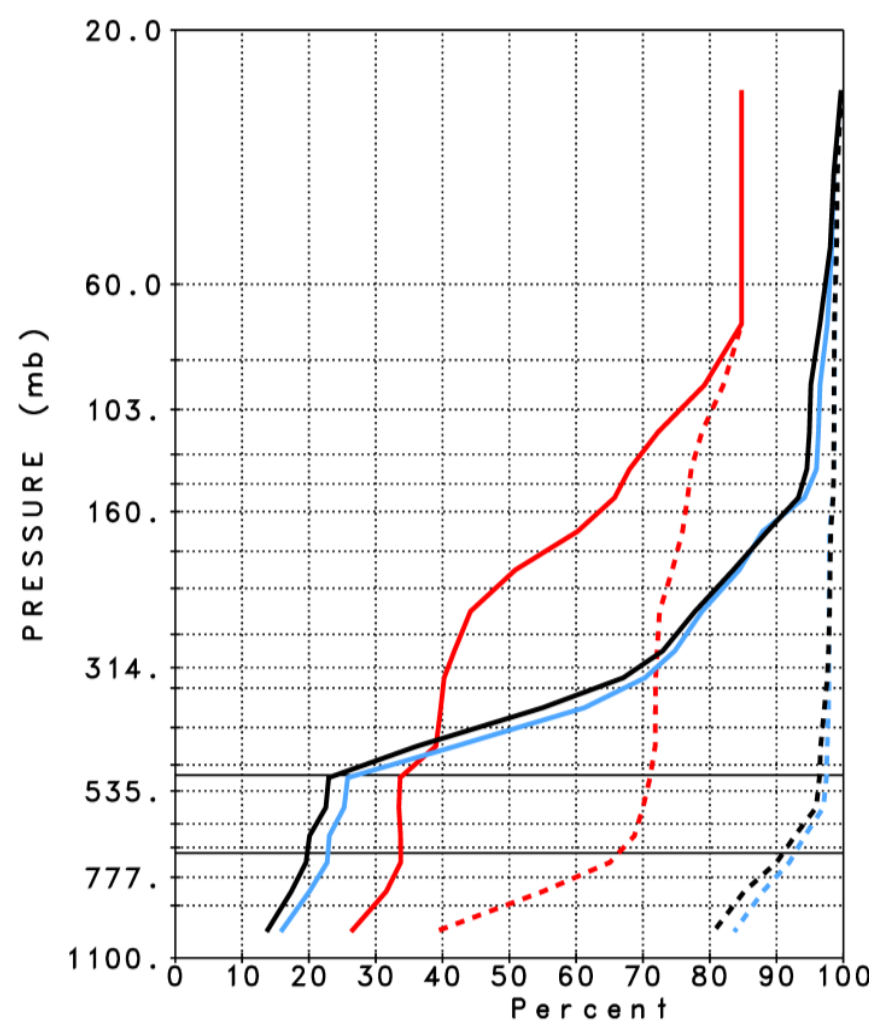

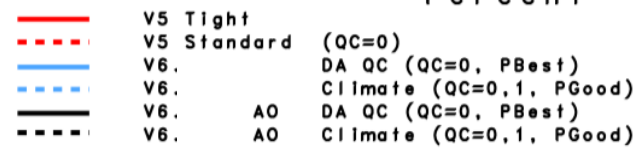

$1 \mathrm{~km}$ Layer Mean RMS ( $\left.{ }^{\circ} \mathrm{K}\right)$

Differences from ECMWF

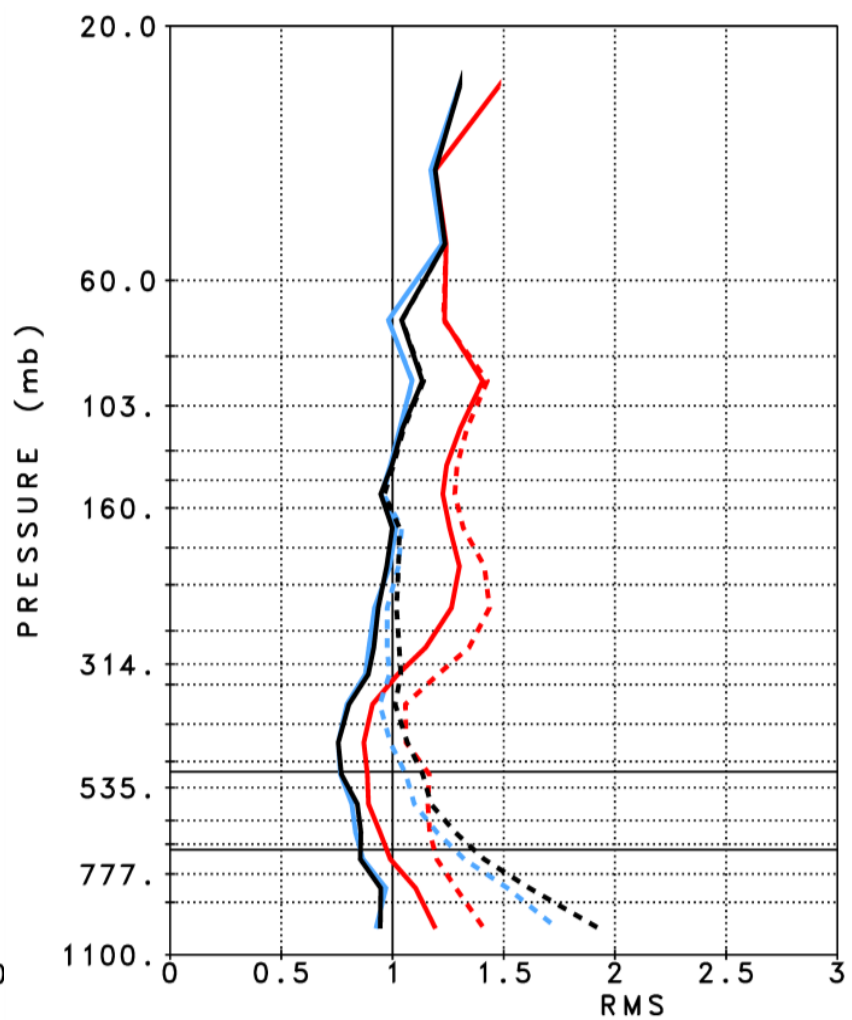

-.-- vi tight

---. V5 Standard $(\mathrm{OC}=0)$

$\begin{array}{ll}\overline{---} & \text { v6 } \\ \overline{---} & \text { v6 } \\ \text { v6 }\end{array}$
1 km Layer Mean BIAS ( $\left.{ }^{\circ} \mathrm{K}\right)$

Differences from ECMWF

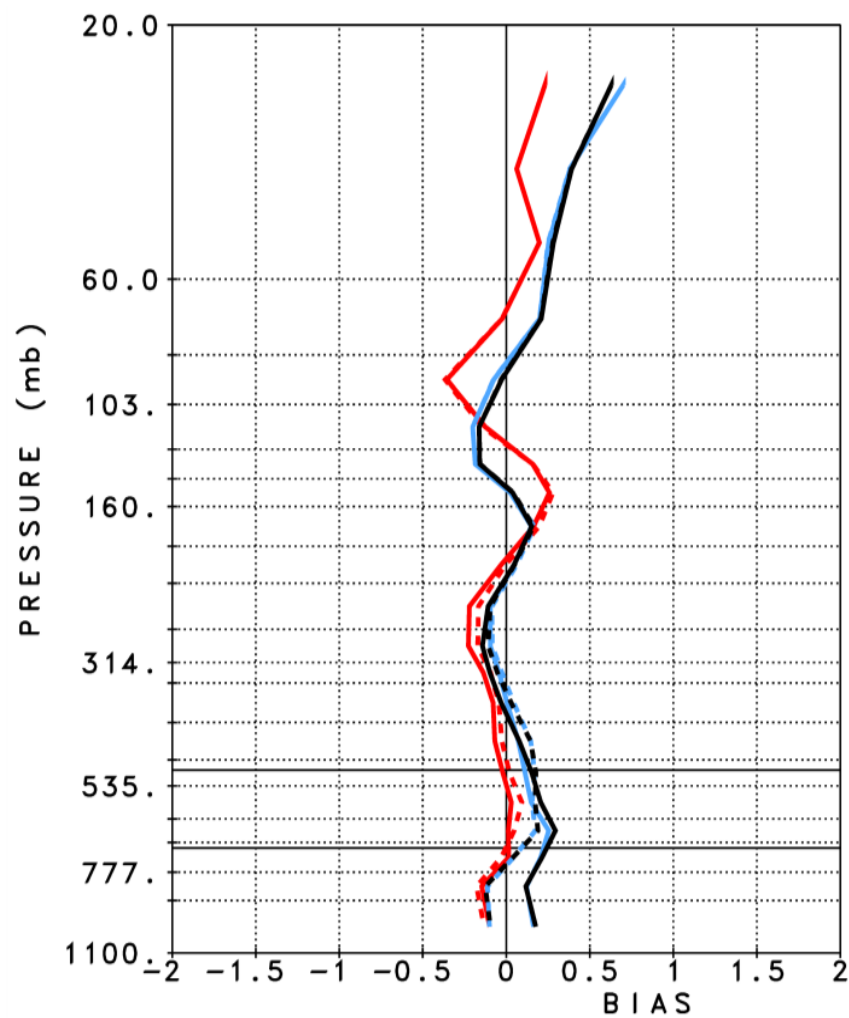

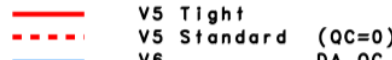

V $V 6$ DA OC ( $Q C=0$, PBest)

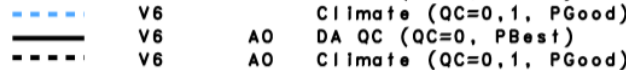

Version-6 T(p) retrievals with DA QC have RMS errors $\leq 1 \mathrm{~K}$ throughout troposphere Version-6 T(p) retrievals with Climate QC have much greater yield than Verison-5 with small biases Differences between Version-6 and Version-6 AO are small 


\section{Global Temperature Layer Mean RMS $\left({ }^{\circ} \mathrm{K}\right)$ Differences from ECMWF}

\section{7-Day Two Common Ensembles}

Final Retrievals

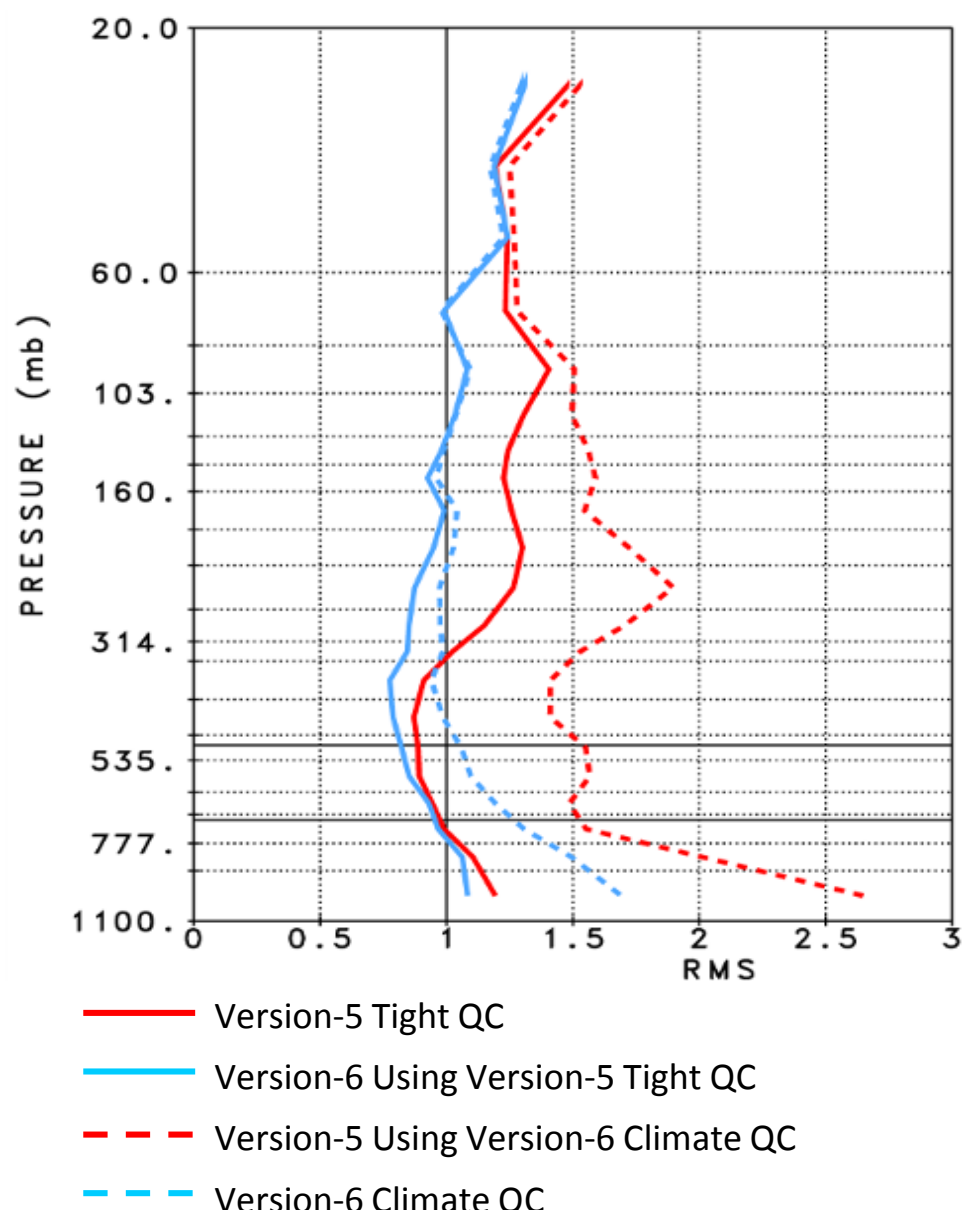

Version-5 Final and First Guess Version-6 Final and First Guess

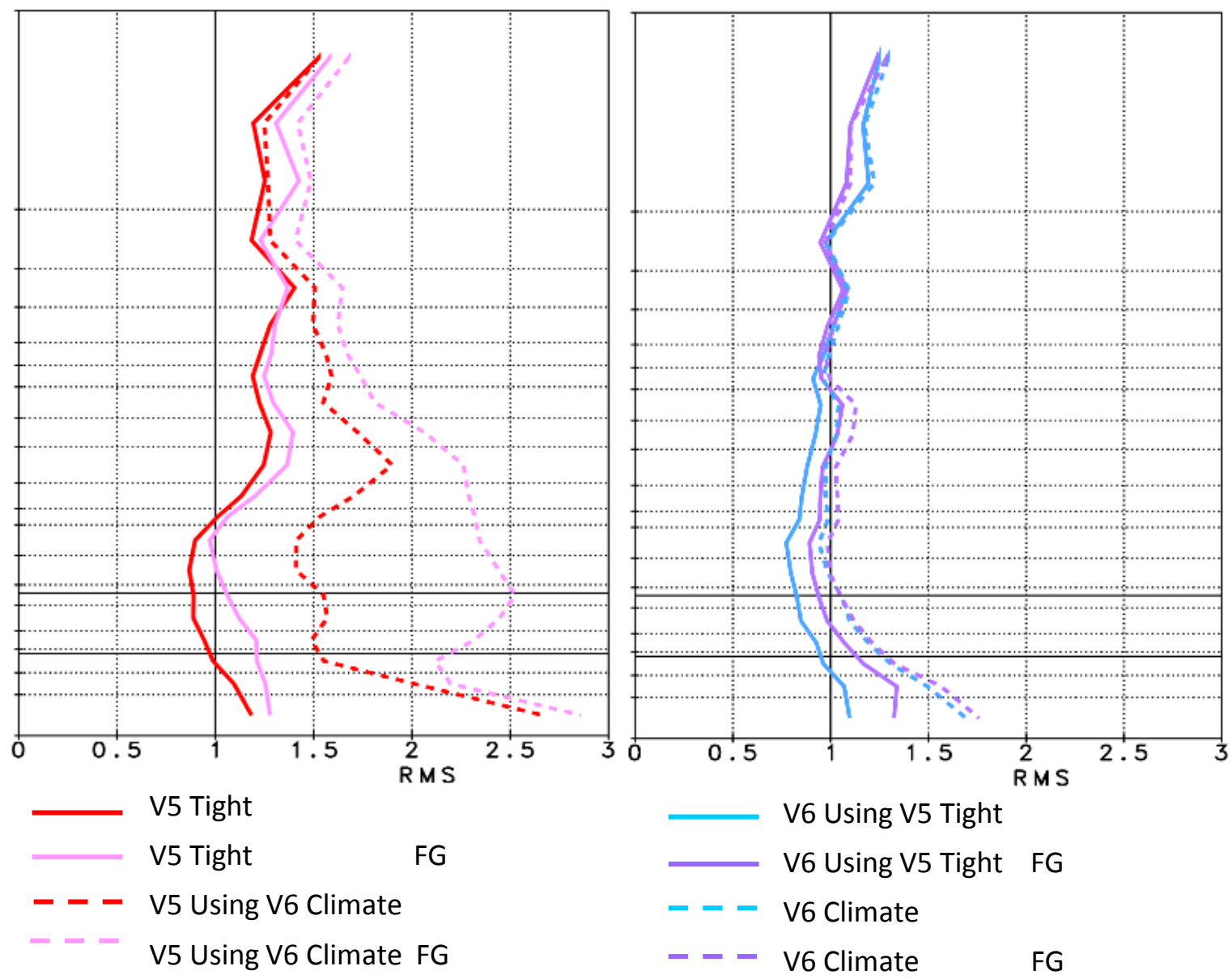

Version- $6 \mathrm{~T}(\mathrm{p})$ retrievals are more accurate than Version-5 on common ensembles, especially for the harder (cloudier) ensembles of cases

Both sets of retrievals improve over their first guesses

The Neural-Net first guess accuracy degrades much more slowly with increasing cloud cover than does regression 


\section{7-Day Mean Statistics Tropospheric Temperature Metric (TTM) and Boundary Layer Metric (BLM)}

Cases in Common Using the Version-5 Tight Ensemble

\begin{tabular}{|c|c|c|c|c|c|c|c|c|c|c|}
\hline & \multicolumn{2}{|c|}{ Global } & \multicolumn{2}{|c|}{ Land $\pm 50^{\circ}$} & \multicolumn{2}{|c|}{ Ocean $\pm 50^{\circ}$} & \multirow{2}{*}{\multicolumn{2}{|c|}{$\frac{\text { Poleward of }}{\underline{50^{\circ} \mathrm{N}}}$}} & \multicolumn{2}{|c|}{ Poleward of $50^{\circ} \mathrm{S}$} \\
\hline & \multirow[t]{2}{*}{ TTM } & \multirow[t]{2}{*}{ BLM } & \multirow[t]{2}{*}{ TTM } & \multirow[t]{2}{*}{$\overline{B L M}$} & \multirow[t]{2}{*}{$\underline{\mathrm{TTM}}$} & \multirow[t]{2}{*}{ BLM } & & & \multirow[t]{2}{*}{$\underline{\text { TTM }}$} & \multirow[t]{2}{*}{ BLM } \\
\hline & & & & & & & TTM & BLM & & \\
\hline Version-5 & 1.10 & 1.29 & 1.19 & 1.71 & 1.04 & 1.13 & 1.14 & 1.50 & 1.31 & 1.76 \\
\hline Version-6.02 & 0.92 & 1.16 & 0.94 & 1.49 & 0.86 & 0.98 & 0.96 & 1.47 & 1.20 & 1.69 \\
\hline
\end{tabular}

\section{Cases in Common Using the Version-6 Climate Ensemble}

\begin{tabular}{|c|c|c|c|c|c|c|c|c|c|c|}
\hline & \multicolumn{2}{|c|}{ Global } & \multicolumn{2}{|c|}{ Land $\pm 50^{\circ}$} & \multicolumn{2}{|c|}{ Ocean $\pm 50^{\circ}$} & \multicolumn{2}{|c|}{ Poleward of $50^{\circ} \mathrm{N}$} & \multirow{2}{*}{\multicolumn{2}{|c|}{$\frac{\text { Poleward of }}{\underline{50^{\circ} \mathrm{S}}}$}} \\
\hline & TTM & BLM & TTM & BLM & TTM & BLM & TTM & BLM & & \\
\hline & & & & & & & & & $\underline{\text { TTM }}$ & BLM \\
\hline Version-5 & 1.67 & 2.57 & 1.82 & 2.78 & 1.65 & 2.48 & 1.53 & 2.39 & 1.72 & 2.72 \\
\hline Version-6 & 1.11 & 1.67 & 1.06 & 1.75 & 1.03 & 1.34 & 1.12 & 1.93 & 1.32 & 2.02 \\
\hline
\end{tabular}

TTM is the average T(p) RMS difference from ECMWF over all $1 \mathrm{~km}$ layers from surface to $100 \mathrm{mb}$ $B L M$ is the average $T(p)$ RMS difference from ECMWF over the lowest six $0.25 \mathrm{~km}$ layers 


\section{rature Trends 7-Day}

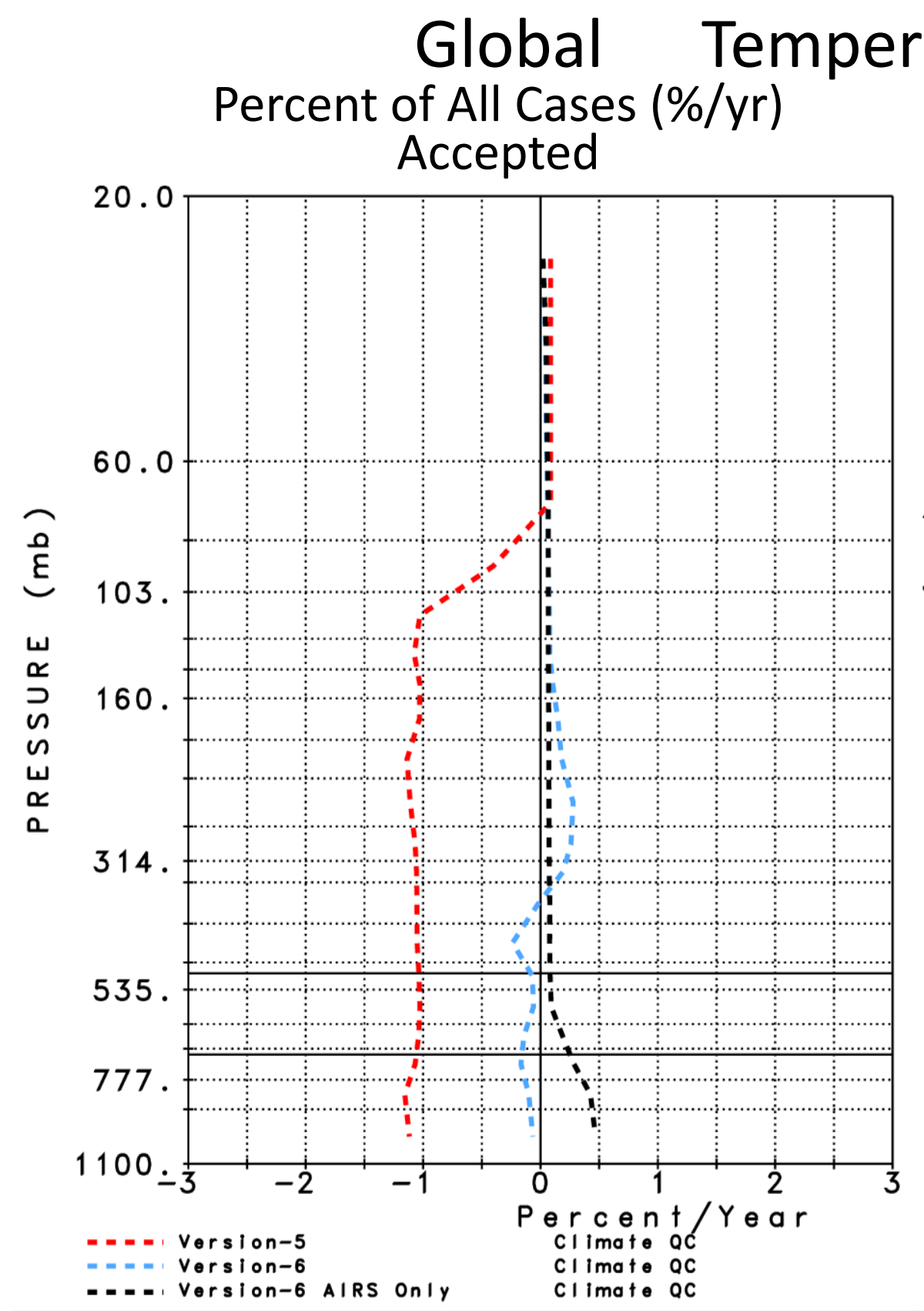

Accepted
Layer Mean BIAS (K/yr)

Differences from ECMWF

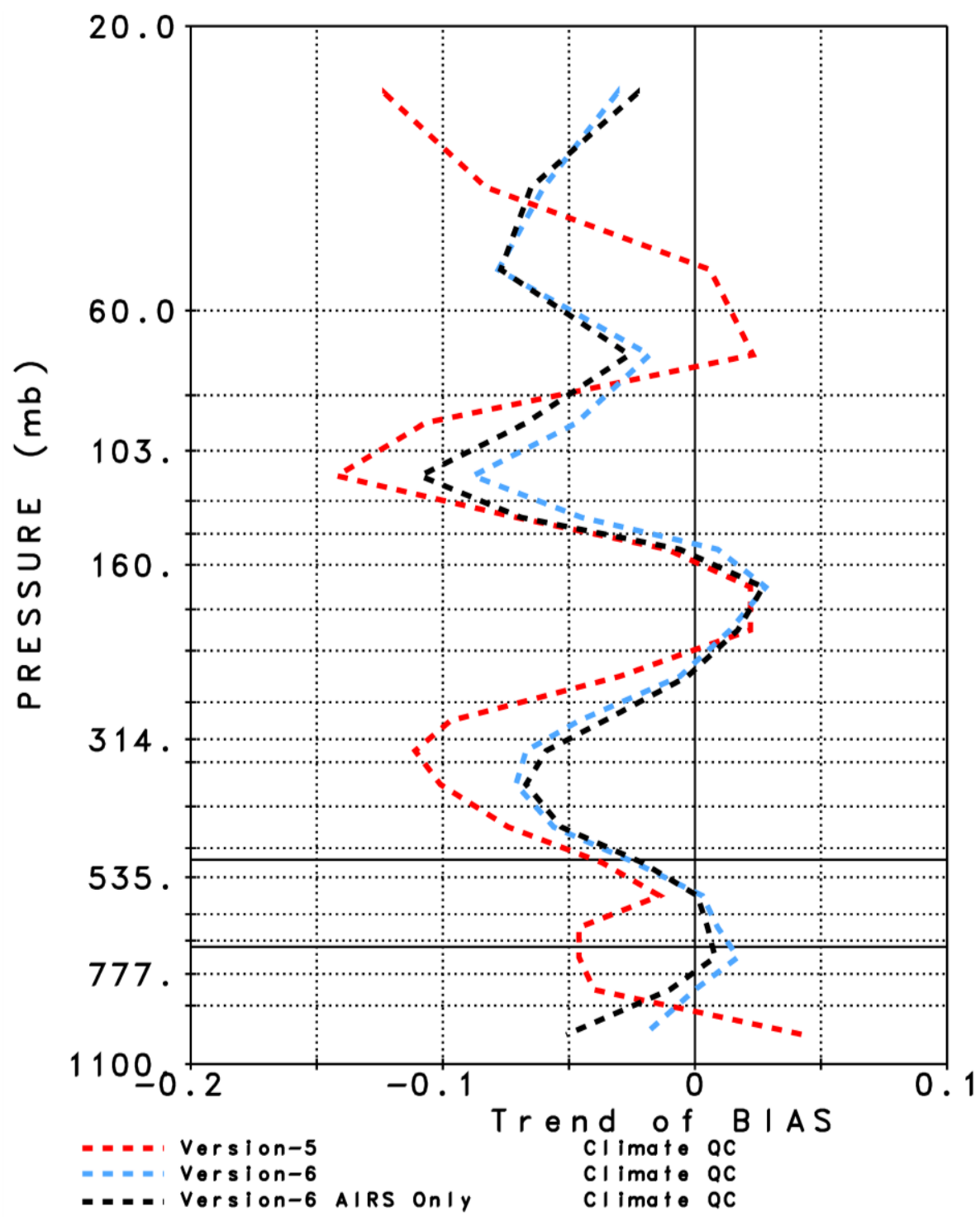

Version- 5 had a significant negative yield trend and negative tropospheric $T(p)$ bias trends compared to ECMWF

These are significantly reduced in Version-6 and Version-6 AO 
AIRS Science Team Version- 6 algorithm determines tropospheric $T(p)$ and $T_{\text {skin }}$ using only shortwave channels $2358 \mathrm{~cm}^{-1}-2664 \mathrm{~cm}^{-1}$. The $15 \mu \mathrm{m}$ tropospheric sounding $\mathrm{CO}_{2}$ channels are used only for cloud clearing (as in Version-5.)

The Version- 6 algorithm also uses a Neural-Net start-up state in place of regression. The Neural-Net guess degrades much more slowly with increasing cloud cover.

These modifications result in:

- Improved soundings of $T(p)$ and SST, day and night.

Improvements are larger with increasing cloud cover.

- Improved retrievals of surface spectral emissivity, day and night.

- $\quad$ The ability to produce AIRS Only retrievals with comparable accuracy to AIRS/AMSU retrievals.

Success of AIRS Only retrievals shows that a Geostationary AIRS-like IR sounder does not require a Geostationary MW sounder, provided it has low noise channels extending to $2500 \mathrm{~cm}^{-1}$. 
NA $3 A$ 